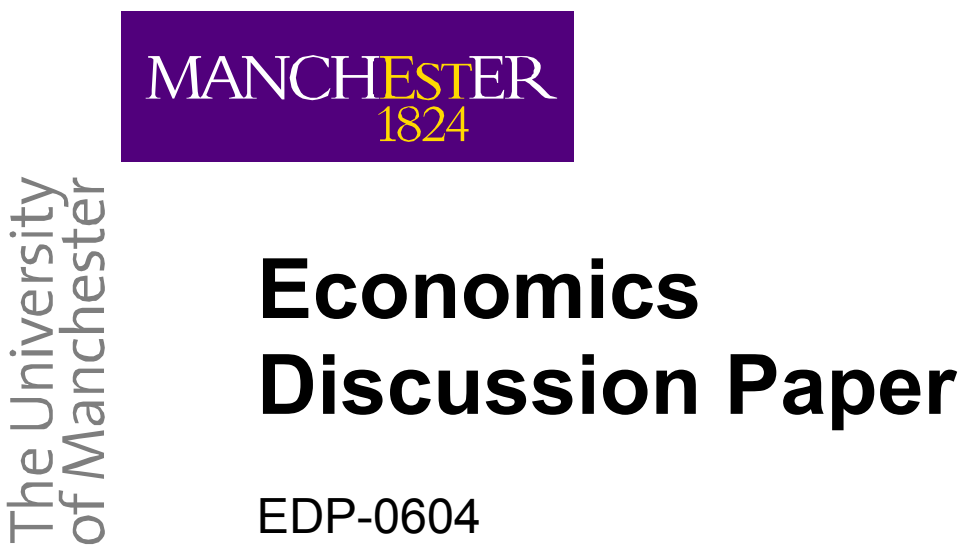

\title{
Minimum Wages and Welfare in a Hotelling Duopsony
}

by

L. Kaas and P. Madden

February 2006

Correspondence email: $\underline{\text { Paul.Madden } @ \text { manchester.ac.uk }}$

School of Social Sciences,

The University of Manchester

Oxford Road

Manchester M13 9PL

United Kingdom 


\title{
MINIMUM WAGES AND WELFARE
}

\section{IN A HOTELLING DUOPSONY}

\author{
Leo Kaas* and Paul Madden**
}

\begin{abstract}
$\underline{\text { Abstract }}$
Two firms choose locations (non-wage job characteristics) on the interval $[0,1]$ prior to announcing wages at which they employ workers who are uniformly distributed over $[0,1]$; the (constant) marginal revenue products of workers may differ. Subgame perfect equilibria of the 2-stage location-wage game are studied under laissez-faire and under a minimum wage regime. UP to a restriction for the existence of pure strategy equilibria, the imposition of a minimum wage is always welfare-improving because of its affect on non-wage job characteristics.
\end{abstract}

JEL classification: D43, E24, J48

Keywords: Hotelling, duopsony, minimum wages

* Department of Economics, University of Konstanz, Box D145, 78457

Konstanz, Germany; email, Leo.Kaas@uni-Konstanz.de.

** School of Social Sciences, University of Manchester, Manchester, M13 9PL, UK; email, Paul.Madden@manchester.ac.uk 


\section{1. $\quad$ INTRODUCTION}

In perfectly competitive labour markets, the imposition of a binding minimum wage leads to reductions in aggregate employment and social welfare. On the other hand it is also well-known that monopsony can lead to increases in aggregate employment and welfare after a minimum wage imposition, a result which has fuelled much debate in the literature (see Manning (2003)). Bhaskar and To (1999) extend the monopsony argument to a differentiated oligopsony; with exogenous, horizontal differentiation of non-wage job characteristics (symmetric locations along a Salop "circular city") welfare improvements from minimum wages emerge again via the aggregate employment channel. Our objective is to demonstrate the existence of a new channel whereby minimum wages can improve welfare in a differentiated oligopsony, namely the choice of non-wage job characteristics. To do this we switch to a Hotelling duopsony ("linear city") model in which firms choose locations (i.e. the non-wage job characteristic) at stage I and wages at stage II of a 2-stage game. Following Bhaskar and To (2003) (where there is no welfare discussion) we also allow the two firms to differ in their inherent efficiency of employing labour (the marginal revenue products differ). Abstracting from all aggregate employment effects we show in detail how the imposition of minimum wages improves social welfare via its impact on the non-wage job characteristics. Indeed (up to a restriction regarding existence of pure strategy equilibria) the imposition of a minimum wage always improves on the laissez-faire outcome. Moreover the mechanism is totally dependant on the changes in non-wage job characteristics; if such changes are impossible (e.g. in a "short-run") minimum wages reduce welfare.

The paper therefore studies the laissez-faire market outcome in a Hotelling duopsony with asymmetric firm efficiency (section 3), and the social optimum in such 
a world (section 3 also). The results of section 3 offer no essential novelty beyond that provided by Ziss (1993) in his completely parallel results for the laissez-faire outcome and social optima in a Hotelling duopoly with asymmetric marginal costs. We go on to study the effect of minimum wages on the market outcome (section 4) and on social welfare (sections 5) with the results claimed above. Section 2 introduces the framework of our analysis and section 6 concludes.

\section{THE FRAMEWORK}

There are 2 firms $(i=0,1)$ producing output from labour at constant marginal revenue product of $\alpha$ for firm 0 and $\beta$ for firm 1 , where $\alpha>\beta$. It maybe that the marginal physical product is higher at firm 0 , or the difference may be caused by firm 0 selling in a more profitable output market than firm 1; for convenience we refer to firm 0 as the efficient firm. The wage offered by firm $i$ is $w_{i}, i=0,1$ and is subject to minimum wage legislation whereby only $w_{i} \geq \bar{w}$ can be chosen; throughout we assume $\bar{w} \in[0, \beta]$ so that the minimum wage does not preclude the operation of either firm.

Each firm also offers a non-wage job characteristic in the interval $[0,1], a \in$ $[0,1]$ for firm 0 and $(1-b) \in[0,1]$ for firm 1 . There is a continuum of workers whose ideal non-wage job characteristics are uniformly distributed over [0, 1]. Taking a job at firm $i$ whose non-wage job characteristic is at a distance $d$ from a worker's ideal provides the worker with job utility $w_{i}-t d^{2}$ where $t>0$ is a parameter. Following d'Aspremont et.al. (1979) we assume that each worker supplies inelastically one unit of labour to the firm that offers the higher job utility, so that the worker whose ideal non-wage characteristic is at $x \in[0,1]$ works for firm 0 if 
$w_{0}-t(x-a)^{2}>w_{1}-t(1-b-x)^{2}$ and at firm 1 if the inequality is reversed, with indifference if there is equality. If $a \neq 1-b$ the solution to the equality is $\tilde{x}=\frac{1}{2}(1-b+a)+\left(w_{0}-w_{1}\right) / 2 t(1-a-b)$, and the labour market shares or employment levels at firm $i=0,1$ will be;

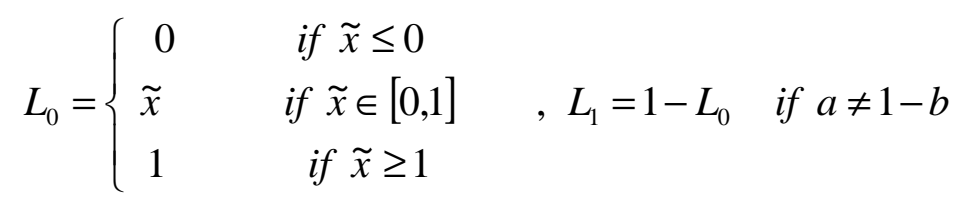

If $a=1-b$, jobs are homogeneous in their non-wage characteristics. In this case, analogous to homogeneous product Bertrand models with asymmetric costs, we assume that the high wage firm gets the whole labour market if $w_{0} \neq w_{1}$, but the efficient firm gets the whole market when $w_{0}=w_{1}$;

$L_{0}=\left\{\begin{array}{l}0 \text { if } w_{0}<w_{1} \\ 1 \text { if } w_{0} \geq w_{1}\end{array} \quad, L_{1}=1-L_{0} \quad\right.$ if $a=1-b$

Firm profits are $\pi_{0}=\left(\alpha-w_{0}\right) L_{0}$ and $\pi_{1}=\left(\beta-w_{1}\right) L_{1}$.

One potential application has the continuum of workers geographical located uniformly along Hotelling's "Main Street", $[0,1]$ on which the firm's locate and workers bear quadratic transport costs of travelling to work. For brevity we use this terminology in the sequel, whose main focus is the two stage game where firms at stage I choose simultaneously locations $a \in[0,1]$ for firm 0 and $1-b \in[0,1]$ for 1 , and offer wages $w_{i} \in W_{\mathrm{i}}, i=0,1$, simultaneously at stage II, employing the forthcoming labour supply and receiving payoffs $=$ profits. We assume $W_{0}=[\bar{w}, A]$ and $W_{1}=$ 
$[\bar{w}, B]$ so that firms cannot offer wages in excess of their marginal revenue product. We do this to avoid some implausible stage II subgame equilibria when firms collocate $(a=1-b)$ again following the homogeneous product Bertrand lead (see Hurter and Lederer (1986) for a discussion).

Although the focus is on the case with $\alpha>\beta$, we also extend results to the case $\alpha=\beta$. With this symmetry (2.2) can be replaced by the analogue of the usual symmetric cost Bertrand assumption:

$$
L_{0}=\left\{\begin{array}{ll}
0 & \text { if } w_{0} \leq w_{1} \\
\frac{1}{2} & \text { if } w_{0}=w_{1} \\
1 & \text { if } w_{0}>w_{1}
\end{array} \quad, L_{1}=1-L_{0} \quad \text { if } a=1-b\right.
$$




\section{TWO BENCHMARKS: LAISSEZ-FAIRE MARKET EQUILIBRIUM AND THE SOCIAL OPTIMUM}

The two main results of this section pertain to the laissez-faire market equilibrium (the subgame perfect equilibrium (SPE) of the 2-stage game when $\bar{w}=0$, Theorem 1) and the social optima (Theorem 2). Both results are due essentially to Ziss (1993), where Proposition 2 equates to Theorem 1 and Proposition 4 is the analogue of Theorem 2. However we include full derivations of our results since the duopsony setting and notation is quite different from Ziss's (1993) duopoloy, and since we use our supporting lemmas to build the minimum wage analysis of sections 4 and 5. We denote $\delta=(\alpha-\beta) / t$ as a measure of the efficiency differential between the firms.

Theorem 1 If $\delta \in\left(0, \delta^{*}\right]$ where $\delta^{*}=6-3 \sqrt{3} \cong 0.81$ and if $\bar{w}=0$, the unique (up to symmetry) pure strategy SPE outcome has maximum location differentiation of the firms ( $a=b=0$ or 1$)$ and the following wages, market shares and profits;

$$
\begin{aligned}
w_{0}^{* *}=\frac{2}{3} \alpha+\frac{1}{3} \beta-t & w_{1}^{* *}=\frac{1}{3} \alpha+\frac{2}{3} \beta-t \\
L_{0}^{* *}=\frac{1}{2}+\frac{1}{6} \delta & L_{1}^{* *}=\frac{1}{2}-\frac{1}{6} \delta \\
\Pi_{0}^{* *}=\frac{1}{18} t(3+\delta)^{2} & \Pi_{1}^{* *}=\frac{1}{18} t(3-\delta)^{2}
\end{aligned}
$$

The following lemmas 3.1, 3.2 and 3.3 (proofs for 3.1 and 3.3 are found in the Appendix) provide a proof of Theorem 1. We first describe the best responses and Nash equilibrium (NE) in stage II subgames at arbitrary locations $(a, b) \in[0,1]^{2}$. Let $S=\left\{(a, b) \in[0,1]^{2}: a+b<1\right\}, \quad H=\left\{(a, b) \in[0,1]^{2}: a+b=1\right\} \quad$ and $T=\{(a, b) \in S: \delta<(1-a-b)(3-a+b)\}$. Note that the subgame at $(a, b) \in S$ has, from symmetry, the same outcome as that at $(1-a, 1-b) \in[0,1]^{2}$, so description of subgame NE for $(a, b) \in S \cup H$ suffices. Note also that when $\delta \leq 3, T$ is a nonempty convex subset of $S$, and has an upper boundary in $S$ which is downward sloping with 
intercepts $a=0, b=\bar{b}=\sqrt{4-\delta}-1$ and $b=0, a=\bar{a}=2-\sqrt{1+\delta} . T$ is the subset of locations where the inefficient firm gets positive market share in the wage subgame, part of the following lemma 3.1

\section{Lemma 3.1}

(a) For a wage subgame with location $(a, b) \in S$, (i) - (iii) describe firm 0's best responses and (iv) - (vi) those of firm 1 , where $\gamma_{0}=\beta-t(1-a-b)(3+a-b)$,

$$
\begin{aligned}
& \Lambda_{0}=\beta+t(1-a-b)(1-a+b), \gamma_{1}=\alpha-t(1-a-b)(3-a+b), \\
& \Lambda_{1}=\alpha+t(1-a-b)(1-b+a) \text {; } \\
& \text { (i) } \quad w_{0}=w_{1}+t\left[(1-a)^{2}-b^{2}\right] \quad \text { if } w_{1}<\gamma_{1} \\
& \text { (ii) } \quad w_{0}=\frac{1}{2}\left\{\alpha+w_{1}-t\left[(1-b)^{2}-a^{2}\right]\right\} \quad \text { if } w_{1} \in\left[\gamma_{1}, \Lambda_{1}\right) \\
& \text { (iii) } \quad w_{0}=[0, \alpha] \quad \text { if } \Lambda_{1} \leq w_{1} \\
& \text { (iv) } \quad w_{1}=w_{0}+t\left[(1-b)^{2}-a^{2}\right] \quad \text { if } w_{0}<\gamma_{0} \\
& \text { (v) } \quad w_{1}=\frac{1}{2}\left\{\beta+w_{0}-t\left[(1-a)^{2}-b^{2}\right]\right\} \quad \text { if } w_{0} \in\left[\gamma_{0}, \Lambda_{0}\right) \\
& \text { (vi) } \quad w_{1}=[0, \beta] \quad \text { if } \Lambda_{0} \leq w_{0}
\end{aligned}
$$

(b) For a wage subgame with location $(a, b) \in T$, the unique Nash equilibrium wages, market shares and profits are;

$$
\begin{aligned}
& w_{0}^{*}(a, b)=\frac{2}{3} \alpha+\frac{1}{3} \beta-\frac{1}{3} t(1-a-b)(3+a-b) \quad, \quad w_{1}^{*}=\frac{1}{3} \alpha+\frac{2}{3} \beta-\frac{1}{3} t(1-a-b)(3-a+b) \\
& L_{0}^{*}(a, b)=\frac{1}{6}(3+a-b)+\frac{\delta}{6(1-a-b)}, \quad L_{1}^{*}(a, b)=\frac{1}{6}(3-a+b)-\frac{\delta}{6(1-a-b)} \\
& \Pi_{0}^{*}(a, b)=\frac{1}{18} t(1-a-b)\left[3+a-b+\frac{\delta}{1-a-b}\right]^{2}, \Pi_{1}^{*}(a, b)=\frac{1}{18} t(1-a-b)\left[3-a+b-\frac{\delta}{1-a-b}\right]^{2}
\end{aligned}
$$

(c) For a wage subgame with location $(a, b) \in(S \cup H) / T$, the unique Nash equilibrium wages, market shares and profits are;

$$
w_{0}^{*}(a, b)=\beta+t\left[(1-a)^{2}-b^{2}\right] \quad w_{1}^{*}(a, b)=\beta
$$




$$
\begin{array}{lrl}
L_{0}^{*}(a, b)=1 & L_{1}^{*}(a, b)=0 \\
\Pi_{0}^{*}(a, b)=\alpha-\beta-t\left[(1-a)^{2}-b^{2}\right] & \Pi_{1}^{*}(a, b)=0
\end{array}
$$

The efficient firm thus sets higher wages in subgames anywhere on $S / T$, and also on $T_{0}=\left\{(a, b) \in T: w_{0}^{*}(a, b)>w_{1}^{*}(a, b)\right\}=\{(a, b) \in T: \delta>2(1-a-b)(a-b)\}$, whereas on $T_{1}=\{(a, b) \in T: \delta<2(1-a-b)(a-b)\}$ firm 1's wage is higher. $T_{1}$ is non-empty iff $\delta<\frac{1}{2}$, illustrated in figure 3.1 .

Figure 3.1 here

When $(a, b) \in T_{0}$ for instance, figure 3.2 shows (as $\mathrm{BR}_{0}, \mathrm{BR}_{1}$ ) the best responses generating the subgame NE(shown with $\left.\beta>\alpha-t\left[(1-b)^{2}-a^{2}\right]\right)$.

Figure 3.2 here

To find SPE we need the NE of the "reduced form" stage I location game where firm 0 chooses $a \in[0,1]$, firm 1 chooses $b \in[0,1]$ and payoffs are given by $\Pi_{i}^{*}(a, b)$ in lemma 3.1. These payoffs are continuous functions. We look first at the "constrained best response" of the inefficient firm in this game, which solves:

$\max _{b} \Pi_{1}^{*}(a, b)$ s.t. $0 \leq b \leq 1-a$. We denote this solution $\psi_{1}(a)$, and $\tilde{\Pi}_{1}(a)$ are the resulting profits.

For $a \in[\bar{a}, 1], \Pi_{1}^{*}(a, b)=0$ for all $b \in[0,1-a]$, so $\psi_{1}(a)=[0,1-a]$ and $\tilde{\Pi}_{1}(a)=0$. For $a \in[0, \bar{a})$, firm 1 can attain positive profit only by choosing $b$ so that $(a, b) \in T$, but then, from lemma 3.1(b);

$$
\partial \Pi_{1}^{*} / \partial b=\frac{1}{18} t(3-a+b-\delta /(1-a-b))(-1-a-3 b-\delta /(1-a-b))<0
$$

Thus $\psi_{1}(a)=0$ and $\tilde{\Pi}_{1}(a)=\Pi_{1}^{*}(a, 0)$ for $a \in[0, \bar{a})$, completing the description of firm 1's constrained best responses. The function $\widetilde{\Pi}_{1}(a)$ thus defined is easily seen to be continuous, strictly decreasing on $[0, \bar{a})$ and constant at 0 on $[\bar{a}, 1]$. 
In firm 1's unconstrained best response problem, it can also choose $b \in[1-a, 1]$. From symmetry the maximum attainable profit over this $b$ interval is $\tilde{\Pi}_{1}(1-a)$, and the unconstrained best response profit for firm 1 is $\max \left[\tilde{\Pi}_{1}(a)\right.$, $\left.\tilde{\Pi}_{1}(1-a)\right]$ attained at the best responses $\psi_{1}(a)$ if $\tilde{\Pi}_{1}(a)>\tilde{\Pi}_{1}(1-a), 1-\psi_{1}(1-a)$ if $\tilde{\Pi}_{1}(1-a)>\tilde{\Pi}_{1}(a) \quad$ and $\quad$ at $\quad\left\{\psi_{1}(a), 1-\psi_{1}(1-a)\right\}$ if $\tilde{\Pi}_{1}(a)=\tilde{\Pi}_{1}(1-a) . \quad$ When $\delta<5 / 4, \bar{a}>\frac{1}{2}$, which proves, given the earlier monotonicity properties of $\tilde{\Pi}_{1}(a)$ :

Lemma 3.2 The best response of firm 1 in the reduced form stage I game when $\delta \in(0,5 / 4)$, is $b=0$ if $a<\frac{1}{2}, b=1$ if $a>\frac{1}{2}$ and $b=\{0,1\}$ if $a=\frac{1}{2}$.

When $\delta<5 / 4$ the inefficient firm simply locates as far as possible from the efficient firm. By contrast the efficient firm has a possible incentive to co-locate with the inefficient rival since it then (anywhere along $H$ ) attains positive profits of $\alpha-\beta$. The same "centifugal" force that drives the inefficient firm to maximum distance from firm 0 , also affects firm 0 's decision, but this force is minimized when firm 1 is at the centre of $[0,1]$ when the maximum distance is smallest. Thus one might expect that firm 0 will want to co-locate when $b$ is near $1 / 2$, moving as far away as possible when $b$ is sufficiently far from the centre. This intuition is borne out in the following precise statements.

Lemma 3.3 The best response of firm 0 in the reduced form stage I game is;

(a) for $\delta \in(0,1 / 4), a=0$ if $b<\frac{1}{2}, a=1$ if $b>\frac{1}{2}, a=\{0,1\}$ if $b=\frac{1}{2}$

(b) for $\delta \in\left[1 / 4, \delta^{*}\right]$, there is a strictly decreasing function $b(\delta)$ with $b\left(\frac{1}{4}\right)=\frac{1}{2}$,

$$
\begin{aligned}
& b\left(\delta^{*}\right)=0 \text { such that } a=0 \text { if } b<b(\delta), a=\{0,1-b\} \text { if } b=b(\delta), a=1-b \text { if } \\
& b \in(b(\delta), 1-b(\delta)), a=\{1-b, 1\} \text { if } b=1-b(\delta) \text { and } a=1 \text { if } b>1-b(\delta)
\end{aligned}
$$

Lemmas 3.2 and 3.3(a) and (b) complete the proof of Theorem 1. 
Finally when $\delta=0$, so $\alpha=\beta$ and $T=S$ the statements in Lemma 3.1(a) and (b) remain valid. The remaining locations in Lemma 3.1(c) are now only the co-location set $H$. On this set the wage subgames are homogeneous Bertrand games with constant, symmetric marginal revenue products, and there is now no reason to assume that firm 0 will take the whole market when wages are equal; the usual assumption is that firms then share the market equally, (2.3) rather than (2.2). Either way the wage subgame payoffs are uniquely 0 for both firms, as in Lemma 3.1(c). So the reduced form stage I game payoffs continue as in Lemma 3.1(b) and (c). Lemma 3.2(a) continues to provide firm 1 best responses in the reduced form game, but firm 0 now has a symmetric response - both firms wish to get as far away as possible from the rival, reproducing the exact d'Aspremont et.al. (1979) parallel:

Corollary to Theorem 1 The statement in Theorem 1 remains true with $\delta=0$ and $\alpha=\beta$.

Our second benchmark is the social optimum. A planner now locates the firms 0 and 1 at $a, 1-b \in[0,1]$ respectively, and dictates the subsets of workers who will work at the 2 firms. We continue to assume that all workers will be employed, the worker located at $x \in[0,1]$ generating surplus $\alpha-t(x-a)^{2}$ at firm 0 and $\beta-t(1-b-x)^{2}$ at firm 1. Without loss of generality $a \leq 1-b$ and, given such $a, b$ it will be socially optimal for some subset $[0, \hat{x}] \subset[0,1]$ to work at 0 and for $(\hat{x}, 1]$ to work at 1 . Social welfare is then the aggregate surplus:

$$
\begin{aligned}
S W(a, b, \hat{x}) & =\alpha \hat{x}+\beta(1-\hat{x})-t \int_{0}^{\hat{x}}(x-a)^{2} d x-t \int_{\hat{x}}^{1}(1-b-x)^{2} d x \\
& =\alpha \hat{x}+\beta(1-\hat{x})-\frac{1}{3} t\left[a^{3}+b^{3}+(\hat{x}-a)^{3}+(1-b-\hat{x})^{3}\right]
\end{aligned}
$$


Given $(a, b)$ the socially optimal $\hat{x}, \hat{x}(a, b)$, equates $\alpha-t(\hat{x}-a)^{2}$ to $\beta-t(1-b-\hat{x})^{2}$ if the resulting $\hat{x} \in[0,1]$, otherwise $\hat{x}=1$. Hence;

$$
\hat{x}(a, b)=\left\{\begin{array}{cc}
\delta / 2(1-b-a)+\frac{1}{2}(1-b+a) & \text { if } \delta \leq(1-a)^{2}-b^{2} \\
1 & \text { if } \delta \geq(1-a)^{2}-b^{2}
\end{array}\right.
$$

Substituting the top branch here into the SW formula and writing $\ell=1-a-b$ produces the function;

$$
f(a, b)=\frac{1}{2}(\alpha+\beta)+\frac{1}{2} \delta^{2} t \ell^{-1}+\frac{1}{2} \delta t(a-b)-\frac{1}{3} t\left\{a^{3}+b^{3}+\frac{1}{8}\left(\delta \ell^{-1}+\ell\right)^{3}+\frac{1}{8}\left(\ell-\delta \ell^{-1}\right)^{3}\right\}
$$

Similar substitution of the bottom branch produces;

$$
g(a, b)=\alpha-\frac{1}{3} t\left[a^{3}+(1-a)^{3}\right]
$$

Hence the maximum social welfare attainable at locations $(a, b) \in S \cup H$ is;

$$
S W(a, b, \hat{x}(a, b))=\left\{\begin{array}{lll}
f(a, b) & \text { if } & \delta \leq(1-a)^{2}-b^{2} \\
g(a, b) & \text { if } & \delta \geq(1-a)^{2}-b^{2}
\end{array}\right.
$$

Theorem 2 Suppose, without loss of generality, that $a \leq 1-b$.

(a) If $\delta \in\left[0, \frac{1}{4}\right)$ the socially optimal location is $a^{0}=\frac{1}{4}+\delta, b^{0}=\frac{1}{4}-\delta$ with and market shares $L_{0}^{0}=\frac{1}{2}+2 \delta, L_{1}^{0}=\frac{1}{2}-2 \delta$

(b) If $\delta \geq \frac{1}{4} \quad$ the socially optimal locations are $a^{0}=\frac{1}{2}, b^{0} \in\left[0, \frac{1}{2}\right]$ with $S W^{0}=\alpha-\frac{1}{12} t$ and market shares $L_{0}^{0}=1, L_{1}^{0}=0$

The proof of Theorem 2 is given in the appendix, and is somewhat different from that of Ziss (1993), avoiding the use of Lagrangeans and the somewhat tricky issue of appropriate concavity of the social welfare function. When $\delta=0$, the socially optimal locations are at the quartiles of the location space, and firms have equal market share, as in d'Aspremont et al (1979). As $\delta$ increases from 0 to $\frac{1}{4}$ the efficient firm is moved towards the centre, the distance between firms staying at $\frac{1}{2}$ and the 
efficient market share increasing from $\frac{1}{2}$ to 1 . For $\delta$ beyond $\frac{1}{4}$ the efficient firm stays at the centre, taking the whole market. Note that Ziss (1993) assumes a small positive set-up cost, so the inefficient firm is not set up when $\delta \geq 1 / 4$. In contrast when $\delta=0$ the market equilibrium moves the firms to opposite extremes of the location space, with equal market share. In this limit, again as in d'Aspremont et al (1979), the market outcome provides socially correct market shares, but too much location differentiation. As $\delta$ increases from 0 to $\delta^{*}$, the market continues to provide too much differentiation, but also allows too much market share for the inefficient firm. 


\section{MINIMUM WAGES AND MARKET EQUILIBRIUM}

Now $\bar{w}>0$. To find the effect on market equilibrium (SPE of the 2 -stage game) we first identify the effect of the minimum wage on the stage II wage subgames at arbitrary stage I locations. We use the notation $\bar{w}_{0}(a, b)=\alpha-t\left[(1-b)^{2}-a^{2}\right]$ and $\bar{w}_{1}(a, b)=\beta-t\left[(1-a)^{2}-b^{2}\right]$.

Lemma 4.1 (a) For wage subgames with locations $(a, b) \in T$ and minimum wage $\bar{w} \in(0, \beta]$, the unique Nash equilibrium wages, prices and profits are;

(A) the laissez-faire values described in lemma 3.1(b) iff $\bar{w} \leq \min \left[w_{0}^{*}(a, b), w_{1}^{*}(a, b)\right]$

(B) $w_{0}=w_{1}=\bar{w}, L_{0}=\frac{1}{2}(1-b+a), L_{1}=1-L_{0}, \Pi_{0}=\frac{1}{2}(\alpha-\bar{w})(1-b+a)$,

$\Pi_{1}=\frac{1}{2}(\beta-\bar{w})(1+b-a)$ iff $\bar{w} \geq \max \left[\bar{w}_{0}(a, b), \bar{w}_{1}(a, b)\right]$

(C) $\quad w_{0}=\frac{1}{2}\left\{\alpha+\bar{w}-t\left[(1-b)^{2}-a^{2}\right]\right\}, \quad w_{1}=\bar{w}$,

$L_{0}=\left\{\alpha-\bar{w}+t\left[(1-b)^{2}-a^{2}\right]\right\} / 4 t(1-a-b)=1-L_{1}$,

$\Pi_{0}=\left\{\alpha-\bar{w}+t\left[(1-b)^{2}-a^{2}\right]\right\}^{2} / 8 t(1-a-b), \Pi_{1}=(\beta-\bar{w}) L_{1}$,

iff $\bar{w}_{0}(a, b) \geq \bar{w} \geq w_{1}^{*}(a, b)$

(D) $\quad w_{0}=\bar{w}, w_{1}=\frac{1}{2}\left\{\beta+\bar{w}-t\left[(1-a)^{2}-b^{2}\right]\right\}$

$L_{0}=1-L_{1} \quad, \quad L_{1}=\left\{\beta-\bar{w}+t\left[(1-a)^{2}-b^{2}\right]\right\} / 4 t(1-a-b)$

$\Pi_{0}=(\alpha-\bar{w}) L_{0} \quad, \quad \Pi_{1}=\left\{\beta-\bar{w}+t\left[(1-a)^{2}-b^{2}\right]\right\}^{2} / 8 t(1-a-b)$

iff $\bar{w}_{1}(a, b) \geq \bar{w} \geq w_{0}^{*}(a, b)$

(b) For wage subgames with locations $(a, b) \in(S \cup H) \backslash T$ and minimum wages $\bar{w} \in(0, \beta]$, the unique Nash equilibrium wages, market shares and profits are the laissez-fiare values described in Lemma 3.1(c). 


\section{$\underline{\text { Proof of Lemma } 4.1}$}

For $(a, b) \in S$, Lemma 3.1(a) and the quasi-concavity of $\pi_{i}$ as a function of $w_{i}$ noted in its proof ensure that the best responses of firm 0 are described by (i) - (iii) below, and those of firm 1 by (iv)-(vi):
(i) $\quad w_{0}=\max \left[\bar{w}, w_{1}+t\left[(1-a)^{2}-b^{2}\right]\right]$
if $w_{1}<\gamma_{1}$
(ii)

$$
w_{0}=\max \left[\bar{w}, \frac{1}{2}\left\{\alpha+w_{1}-t\left[(1-b)^{2}-a^{2}\right]\right\}\right] \quad \text { if } w_{1} \in\left[\gamma_{1}, \Lambda_{1}\right)
$$
(iii) $\quad w_{0}=[\bar{w}, \alpha]$
if $\Lambda_{1} \leq w_{1}$
(iv)

$$
w_{1}=\max \left[\bar{w}, w_{0}+t\left[(1-b)^{2}-a^{2}\right]\right] \quad \text { if } w_{0}<\gamma_{0}
$$

$$
w_{1}=\max \left[\bar{w}, \frac{1}{2}\left\{\beta+w_{0}-t\left[(1-a)^{2}-b^{2}\right]\right\}\right] \quad \text { if } w_{0} \in\left[\gamma_{0}, \Lambda_{0}\right)
$$
(vi) $\quad w_{1}=[\bar{w}, \beta]$
if $\Lambda_{0} \leq w_{0}$

Thus NE for subgames with $(a, b) \in S$ and $\bar{w} \in[0, \beta]$ correspond to solutions for $w_{0} \in[\bar{w}, \alpha], w_{1} \in[\bar{w}, \beta]$ of one of (i)-(iii) coupled with one of (iv)-(vi).

(a) Suppose $(a, b) \in T$. Comparing the above best responses (i)-(vi) with those of Lemma 3.1(a) it is immediate that the laissez-faire outcomes continue as NE iff $\bar{w} \leq \min \left[w_{0}^{*}(a, b), w_{1}^{*}(a, b)\right]$, completing the proof of $(\mathrm{A})$.

Solutions with $w_{0}=w_{1}=\bar{w}$ can be generated by the (ii)/(v) pairing iff:

$$
\begin{aligned}
& \text { (1) } \bar{w} \geq \bar{w}_{0}(a, b)=\alpha-t\left[(1-b)^{2}-a^{2}\right] \quad \text { (2) } \bar{w} \geq \bar{w}_{1}(a, b)=\beta-t\left[(1-a)^{2}-b^{2}\right] \\
& \text { (3) } \bar{w} \geq \gamma_{1}=\alpha-t(1-a-b)(3-a+b) \text { (4) } \bar{w} \geq \gamma_{0}=\beta-t(1-a-b)(3+a-b)
\end{aligned}
$$

But (1) $\Rightarrow(3)$ and (2) $\Rightarrow(4)$. Thus (ii)/(v) produce NE with $w_{0}=w_{1}=\bar{w}$ (and the corresponding market shares and profits in (B)) iff (1) and (2) hold. It is straight forward to check that no pairings produce other NE with $w_{0}=w_{1}=\bar{w}$, completing the proof of (B). 
The (ii)/(v) pairing produces solutions with $w_{0} \geq \bar{w}=w_{1}$ iff

$$
\begin{aligned}
& w_{0}=\frac{1}{2}\left\{\alpha+\bar{w}-t\left[(1-b)^{2}-a^{2}\right]\right\}, w_{1}=\bar{w} \text { and } \\
& \text { (5) } \bar{w} \leq \bar{w}_{0}(a, b)=\alpha-t\left[(1-b)^{2}-a^{2}\right] \quad(6) \bar{w} \geq \gamma_{1}=\alpha-t(1-a-b)(3-a+b) \\
& \text { (7) } \bar{w} \geq \frac{1}{2}\left\{\beta+w_{0}-t\left[(1-a)^{2}-b^{2}\right]\right\} \text { (8) } \Lambda_{0}>w_{0} \geq \gamma_{0}=\beta-t(1-a-b)(3+a-b)
\end{aligned}
$$

Substitution of $\mathrm{w}_{0}$ shows (7) is equivalent to $\bar{w} \geq w_{1}^{*}(a, b)$. For $(a, b) \in T$ the inequalities in (5) and (7) imply those of (6) and (8), so (ii)/(v) produce NE with $w_{0} \geq w_{1}=\bar{w}$ (and market shares and profits of (c)) iff (5) and (7) hold. Again no pairings produce other NE with $w_{0} \geq w_{1}=\bar{w}$, completing (C). The proof of (D) is symmetric to that for $(\mathrm{C})$.

(b) For $(a, b) \in S \backslash H$, the laissez-faire outcomes in Lemma 3.1(c) always continue as NE since for $i=0,1 w_{i}^{*}(a, b) \geq \beta \geq \bar{w}$. It is straightforward to check that no pairings (of (i)-(iii) with (iv)-(vi)) produce any other NE. For $(a, b) \in H$, the argument for this case in Lemma 3.1(c) ensures that the laissez-faire outcome is the unique NE for any $\bar{w} \in[0, \beta]$, completing (b).

Lemma 4.1 shows that there are 4 possible types of wage subgame equilibrium, where the minimum wage is (weakly) binding on (A) neither firm, (B) both firms, (C) the inefficient firm only and (D) the efficient firm only; we label these different types as "type $i$ equlibrium", $i=\mathrm{A}, \mathrm{B}, \mathrm{C}, \mathrm{D}$. For given $\delta$ the relation between locations and wage subgame equilibrium type varies with $\bar{w}$ and is now described.

Notice first that if $\bar{w} \leq w_{1}^{* *}$ then $\bar{w}$ is never strictly binding on either firm in any wage subgame and type A equilibrium occurs at all locations. Thus the laissezfaire SPE continues as in Theorem 1 and its Corollary 1: 
Corollary 2 to Theorem 1 The statements in Theorem 1 and its Corollary 1 remain true for any $\bar{w} \in\left(0, w_{1}^{* *}\right]$

If $\bar{w}>w_{1}^{* * *}$ some wage subgame outcomes (e.g. at $a=b=0$ ) will be affected by the minimum wage. The following discussion is truncated to the assumption that $\delta \in\left[0, \frac{1}{2}\right.$ ) (for brevity, and because qualitatively nothing of further interest emerges outside this range), and for the time being we focus on $\delta \in\left(0, \frac{1}{2}\right)$. The set $T_{1}$ where firm 1 offers the higher wage in laissez-faire wage subgames is then non-empty, and the effect of $\bar{w}>w_{1}^{* *}$ on wage subgames is described by figure 4.1 and lemma 4.2, using the following notation for some critical minimum wages $\bar{w}_{1}, \bar{w}_{2}$ and $\bar{w}_{3}$ (all belong to $\left(w_{1}^{* *}, \beta\right)$ and $\left.\bar{w}_{1}<\bar{w}_{2}<\bar{w}_{3}\right)$;

$\bar{w}_{1}=\alpha-t \quad ; \quad \bar{w}_{2}=\alpha-\frac{1}{2} t(1+\delta+\sqrt{1-2 \delta}) \quad ; \quad \bar{w}_{3}=\delta-\frac{1}{2} t(1+\delta-\sqrt{1-2 \delta})$

When $0<\delta<\frac{1}{2}$ figure 4.1 shows how the equilibrium type varies with location as $\bar{w}$ increases from $w_{1}^{* *}$, and Lemma 4.2 is the formal statement. In the diagram the dotted curve is $\delta=2(1-a-b)(a-b)$, the $\mathrm{A} / \mathrm{C}$ border has $\bar{w}=w_{1}^{*}(a, b)$, the $\mathrm{B} / \mathrm{C}$ border $\bar{w}=\bar{w}_{0}(a, b), \mathrm{A} / \mathrm{D} \bar{w}=w_{0}^{*}(a, b)$ and $\mathrm{B} / \mathrm{D} \bar{w}=\bar{w}_{1}(a, b)$.

Figure 4.1 here

\section{Lemma 4.2 Suppose $0<\delta<0.5$.}

(a) If $w_{1}^{* *} \leq \bar{w}<\bar{w}_{1}$ there is type $\mathrm{C}$ equilibrium if $\bar{w} \geq w_{1}^{*}(a, b)$ and type $\mathrm{A}$ if $\bar{w} \leq w_{1}^{*}(a, b)$

(b) If $\bar{w}_{1} \leq \bar{w} \leq \bar{w}_{2}$ there is type $\mathrm{B}$ equilibrium if $\bar{w} \geq \bar{w}_{0}(a, b)$, type $\mathrm{C}$ if $\bar{w}_{0}(a, b) \geq \bar{w} \geq \bar{w}_{1}^{*}(a, b)$ and type $\mathrm{A}$ if $\bar{w} \leq w_{1}^{*}(a, b)$ 
(c) If $\bar{w}_{2} \leq \bar{w} \leq \bar{w}_{3}$ there is type $\mathrm{B}$ equilibrium if either $w_{0}^{*}(a, b) \geq w_{1}^{*}(a, b)$ and $\bar{w} \geq \bar{w}_{0}(a, b)$, or $w_{0}^{*}(a, b) \leq w_{1}^{*}(a, b)$ and $\bar{w} \geq \bar{w}_{1}(a, b)$, type A equilibrium if either $w_{0}^{*}(a, b) \geq w_{1}^{*}(a, b) \geq \bar{w}$ or $\quad w_{1}^{*}(a, b) \geq \quad w_{0}^{*}(a, b) \geq \bar{w}, \quad$ type $\quad \mathrm{C} \quad$ if $w_{0}^{*}(a, b) \geq w_{1}^{*}(a, b) \quad$ and $\quad \bar{w}_{0}(a, b) \geq \bar{w} \geq w_{1}^{*}(a, b), \quad$ and $\quad$ type $\quad \mathrm{D} \quad$ if $w_{0}^{*}(a, b) \leq w_{1}^{*}(a, b)$ and $\bar{w}_{1}(a, b) \geq \bar{w} \geq w_{0}^{*}(a, b)$.

(d) If $\bar{w}_{3} \leq \bar{w} \leq \beta$ there is a type $\mathrm{B}$ equilibrium if $\bar{w} \geq \bar{w}_{0}(a, b)$, type $\mathrm{C}$ if $\bar{w}_{0}(a, b) \geq \bar{w} \geq w_{1}^{*}(a, b)$ and type $\mathrm{A}$ if $\bar{w} \leq w_{1}^{*}(a, b)$

Proof of Lemma 4.2 For given $\bar{w}$ we consider the following 4 curves in the $(a, b)$ plane:

(1) $\bar{w}=w_{0}^{*}(a, b)$, (2) $\bar{w}=w_{1}^{*}(a, b)$, (3) $\bar{w}=\bar{w}_{0}(a, b)$, (4) $\bar{w}=\bar{w}_{1}(a, b)$. It is easily confirmed that (i) these 4 curves intersect only where $\delta=2(1-a-b)(a-b)$, the dotted curve in Figure 4.1; (ii) all 4 curves are downard sloping in $S$; when $w_{0}^{*}(a, b)>w_{1}^{*}(a, b),(2)$ lies above (3) in $S$; when $w_{1}^{*}(a, b)>w_{0}^{*}(a, b)$, (1) lies above (4) in $S$; the intersection of the 4 curves occurs where $b \leq 0$ and $a>\frac{1}{2}$ if $\bar{w} \geq \bar{w}_{3}$, which produces (d) in Lemma 4.2; the intersection occurs where $a \geq 0$ if $\bar{w}_{2} \leq \bar{w} \leq \bar{w}_{3}$, which produces (c); when $\bar{w} \leq \bar{w}_{2}$ (b) emerges as long as $\bar{w} \geq \bar{w}_{1}$, and (a) is the outcome if $\bar{w} \leq \bar{w}_{1}$

For any $\delta \in\left(0, \frac{1}{2}\right)$ and $\bar{w} \in[0, \beta]$, Lemmas 4.1 and 4.2 define the payoffs in the reduced form stage I game whose NE correspond to the SPE outcomes. Suppressing the dependence on $\delta$ and $\bar{w}$ we denote these payoffs as $\hat{\Pi}_{i}(a, b), i=0,1$, if $\delta$, $\bar{w}$, and $(a, b)$ produce type A equilibrium, $\hat{\Pi}_{i}(a, b)$ is given by Lemma 4.1(A); and similarly 
for type $\mathrm{B}, \mathrm{C}$ and $\mathrm{D}$ equilibrium. The resulting functions $\hat{\Pi}_{i}: S \cup H \rightarrow R_{+}$are continuous, and differentiable almost everywhere.

The nature of the inefficient firm's constrained best responses in the reduced form stage I game (solutions to $\max _{b} \hat{\Pi}_{1}(a, b)$ s.t. $a \leq 1-b$ ) follows from the following properties (i)-(iv) of the derivative $\partial \hat{\Pi}_{1} / \partial b$. First remember that $\hat{\Pi}_{1}(a, b)=\Pi_{1}^{*}(a, b)=0$ for $(a, b) \in(S \cup H) \backslash T \quad$ (a subset of the type A equilibrium domains in figure 4.1), and we restrict attention to $(a, b) \in T$ in (i) - (iv).

(i) At an interior type A (laissez-faire) equilibrium (where $\left.\bar{w}<\min \left[w_{0}^{*}(a, b), w_{1}^{*}(a, b)\right]\right)$, as in section $3, \partial \hat{\Pi}_{1} / \partial b=\partial \Pi_{1}^{*} / \partial b<0$.

(ii) At an interior type $\mathrm{B}$ equilibrium (where either $\bar{w}>\bar{w}_{0}(a, b)$ and $w_{0}^{*}(a, b)>w_{1}^{*}(a, b), \quad$ or $\quad \bar{w}>\bar{w}_{1}(a, b) \quad$ and $\left.\quad w_{1}^{*}(a, b)>w_{0}^{*}(a, b)\right)$, $\partial \hat{\Pi}_{1} / \partial b=\frac{1}{2}(\beta-\bar{w})>0$

(iii) At an interior type $\mathrm{C}$ equilibrium $\left(\bar{w}<\bar{w}_{0}(a, b)\right.$ and $\left.w_{0}^{*}(a, b)>w_{1}^{*}(a, b)\right), \partial \hat{\Pi}_{1} / \partial b=(\beta-\bar{w})\left[t(1-a-b)^{2}-(A-\bar{w})\right] / 4 t(1-a-b)^{2}<0$ if $\bar{w}<\alpha-t(1-a-b)^{2}$, which follows from $\bar{w}<\bar{w}_{0}(a, b)$; thus $\partial \hat{\Pi}_{1} / \partial b<0$.

(iv) At an interior type $\mathrm{D}$ equilibrium $\left(\bar{w}<\bar{w}_{1}(a, b)\right.$ and $\left.w_{1}^{*}(a, b)>w_{0}^{*}(a, b)\right)$, $\partial \hat{\Pi}_{1} / \partial b$ has the sign of $\beta+t(1-a-b)(1-a-3 b)-\bar{w}$ which is positive since $\beta-t(1-a-d)(1-a+b)-\bar{w}>0 ;$ here $\partial \hat{\Pi}_{1} / \partial b>0$.

For $a \in[0,1]$ where $(a, 0) \in T$, firm 1's constrained best response is, with reference to figure 4.1 , either at the point $b>0$ where $(a, b)$ is on the $\mathrm{B} / \mathrm{C}$ border (if there is such a point), or at the point $b>0$ where $(a, b)$ is on the $\mathrm{A} / \mathrm{D}$ border (if there is 
such a point), or at $b=0$ otherwise. And when $(a, 0) \notin T$, any $b \in[0,1-a]$ is a constrained best response, producing zero profit. Let $\varphi_{1}(a)$ denote firm 1's constrained best response (correspondence) where $a \in[0,1]$, suppressing the dependence on $\delta$ and $\bar{w}$, and let $\bar{a}=2-\sqrt{1+\delta}$. Routine calculations produce the following precise statement;

Lemma 4.3 Suppose $\delta \in\left(0, \frac{1}{2}\right)$. For wage subgames with locations $(a, b)$ $\in S \cup H$ and a minimum wage $\bar{w} \in\left[w_{1}^{* *}, \beta\right]$, firm 1's constrained best response, $\phi_{1}(a)$, is:

(a) For $a \in[0, \bar{a}]$;

(i) $\quad \phi_{1}(a)=0$ if $w_{1}^{* *} \leq \bar{w} \leq \bar{w}_{1}$

(ii) $\quad \phi_{1}(a)=0$ for $a \in\left[1-\frac{\alpha-\bar{w}}{t}, \bar{a}\right]$ and $\phi_{1}(a)=1-\sqrt{a^{2}+\frac{\alpha-\bar{w}}{t}}$ for $a \in\left[0, \sqrt{1-\frac{\alpha-\bar{w}}{t}}\right]$, if $\bar{w}_{1} \leq \bar{w} \leq \bar{w}_{2}$

(iii) with $\quad a_{1}=\sqrt{4-(2+\beta-3 \bar{w}) / t}-1 \quad$ and $\quad a_{2}=\frac{1}{2}-\frac{1}{4}\left(2 \frac{\alpha-\bar{w}}{t}-\delta\right)+$

$$
\begin{array}{ll}
\frac{\delta}{2\left(2 \frac{\alpha-\bar{w}}{t}-\delta\right)}, & \phi_{1}(a)=0 \text { for } \\
\phi_{1}(a)=2-\sqrt{(1+a)^{2}+(2 \alpha+\beta-3 \bar{w}) / t} \quad \text { for } a \in\left[a_{2}, a_{1}\right] & \text { and } \\
\phi_{1}(a)=1-\sqrt{a^{2}+\frac{\alpha-\bar{w}}{t}} \text { for } a \in\left[0, a_{2}\right], \text { if } \bar{w}_{2} \leq \bar{w} \leq \bar{w}_{3} & \text { and }
\end{array}
$$

(iv) $\phi_{1}(a)=0$ for $a \in\left[\sqrt{1-\frac{\alpha-\bar{w}}{t}}, \bar{a}\right]$ and $\phi_{1}(a)=1-\sqrt{a^{2}+\frac{\alpha-\bar{w}}{t}}$

for $a \in\left[0, \sqrt{1-\frac{\alpha-\bar{w}}{t}}\right]$, if $\bar{w}_{3} \leq \bar{w} \leq \beta$

(b) for $a \in[\bar{a}, 1], \phi_{1}(a)=[0,1-a]$. 
The graph of firm 1's constrained best response is as follows. If $a \in[\bar{a}, 1]$ in figure 4.1(a)-(d) then this best response (correspondence) graph is the set of $(a, b)$ where $b \in[0,1-a]$, from Lemma 4.3(b). For $a \in[0, \bar{a}]$ in figure 4.1(a) the graph is the set of $(a, b)$ where $b=0$; in figure 4.1(b) the graph follows the path $Y_{1}, Y_{2},(\bar{a}, 0)$; in figure 4.1(c) it follows $Y_{1}, Y_{2}, Y_{3},(\bar{a}, 0)$ and in figure 4.1(d) if follows $Y_{1}, Y_{2}$, $(\bar{a}, 0)$

Note for future reference that a new critical minimum wage value will emerge when, in figure 4.1(c), $\mathrm{Y}_{2}$ has a coordinate of $\frac{1}{2}$. This requires $\delta=2(1-a-b)(a-b)$ and (e.g.) $\bar{w}=w_{0}^{*}(a, b)$ with $a=\frac{1}{2}$, which become $\delta=2\left(\frac{1}{2}-b\right)^{2}$ and $\bar{w}=\alpha-\frac{1}{3} t\left[\delta+\left(\frac{1}{2}-b\right)\left(\frac{7}{2}-b\right)\right]$ producing the value $\bar{w}=\overline{\bar{w}}$ (say) $=$ $\alpha-\frac{1}{2} t(\delta+\sqrt{2 t}) \in\left(\bar{w}_{2}, \bar{w}_{3}\right)$. The reason for the criticality of $\overline{\bar{w}}$ will be clear after:

Lemma 4.4 For any $\delta \in\left(0, \frac{1}{2}\right)$ and $\bar{w} \in\left[w_{1}^{* *}, \beta\right]$, the unconstrained best response of firm 1 in the reduced form stage I game is $\phi_{1}(a)$ if $a<\frac{1}{2}, 1-\phi_{1}(1-a)$ if $a>\frac{1}{2}$ and $\left\{\phi_{1}(a), 1-\phi_{1}(1-a)\right\}$ if $a=\frac{1}{2}$, where $\phi_{1}(a)$ is the constrained best response described in Lemma 4.3.

\section{$\underline{\text { Proof of Lemma } 4.4}$}

Suppressing other arguments, let $\tilde{\tilde{\pi}}_{1}(a)$ denote firm 1's constrained best response profits to $a \in[0,1] \quad \tilde{\tilde{\pi}}_{1}(a)=0$ for $a \in[\bar{a}, 1]$, from Lemma 4.3(b), where $\bar{a}>\frac{1}{2}\left(\right.$ since $\left.\delta<\frac{1}{2}\right)$; also $\tilde{\tilde{\pi}}_{1}(a)$ is a continuous function, form figure 4.1 since $\hat{\pi}_{1}(a, b)$ is a continuous function. We now show that $\tilde{\tilde{\pi}}_{1}(a)$ is a strictly decreasing function on $[0, \bar{a})$; the symmetry arguments used earlier to establish Lemma 3.2 then complete the proof of Lemma 4.4 . 
Along vertical segments of firm 1's constrained best response graph $((0,0)$ to $(\bar{a}, 0))$ in figure $4.1(\mathrm{a}), Y_{2}$ to $(\bar{a}, 0)$ in figures $4.1(\mathrm{~b})$ and $(\mathrm{d}), Y_{3}$ to $(\bar{a}, 0)$ in figure 4.1(c)), $\quad \tilde{\tilde{\pi}}_{1}(a)=\pi_{1}^{*}(a, 0)=\frac{1}{18} t\left(3-a-\frac{\delta}{1-a}\right)^{2}$ and $\partial \tilde{\tilde{\pi}}_{1} / \partial a<0$. Along segments of 1 's constrained best response graph that coincide with the A/D border $\left(Y_{2}\right.$ to $Y_{3}$ in figure $\left.4.1(c)\right) \quad \tilde{\tilde{\pi}}_{1}(a)=\pi_{1}^{*}(a, b(a)) \quad$ where $\quad b(a) \quad$ is defined by $\bar{w}=\bar{w}_{0}^{*}(a, b(a))$ so that $b^{\prime}(a)=-(1+a)(2-b)^{-1} \in(-1,0)$ which ensures $\partial \tilde{\tilde{\pi}}_{1} / \partial a<0$ here also. Finally, along segments of 1's constrained best response graph that coincide with the $\mathrm{B} / \mathrm{C}$ border ( $Y_{1}$ to $Y_{2}$ in figures 4.1(b), (c) and (d)), $\tilde{\tilde{\pi}}_{1}(a)=\frac{1}{2}(\beta-\bar{w})(1+b(a)-a)$ where $\mathrm{b}(\mathrm{a})$ is now defined by $\bar{w}=\bar{w}_{0}(a, b(a))$ so $b^{\prime}(a)=-a(1-b)^{-1}<0$ and again $\partial \tilde{\tilde{\pi}}_{1} / \partial a<0$.

Hence firm 1's unconstrained best response graph follows the constrained graph if $a \in\left[0, \frac{1}{2}\right]$ with a jump at $a=\frac{1}{2}$ so that for $a \in\left[\frac{1}{2}, 1\right]$ the unconstrained graph extends to include the points $(a, b)$ where $1-b$ is a constrained best response to 1- $a$. The candidates for pure strategy SPE of the 2-stage game with minimum wage $\bar{w} \in\left[w_{1}^{* *}, \beta\right]$ are the points on firm 1's unconstrained best response graph. Figure 4.2, illustrates in bold firm 1's unconstrained best response graphs in $S \cup H$; the extension to $[0,1]^{2}$ adds $(1-a, 1-b)$ for each $(a, b) \in S \cup H$ shown.

Figure 4.2 here

Consideration of firm 0's best responses at points on 1's best response graph leads to:

Theorem 3 (a) If $\delta \in\left(0, \frac{1}{2}\right)$ and $\bar{w} \in\left(w_{1}^{* *}, \overline{\bar{w}}\right]$, the unique (up to symmetry) pure strategy SPE outcome has the following locations, wages, market shares and profits:

$$
\begin{aligned}
& (a, b) \text { defined by } w_{1}^{*}(a, 0)=\bar{w} \text { and } b=0, \quad w_{0}=w_{0}^{*}(a, 0)>w_{1}=\bar{w}, \\
& L_{i}=L_{i}^{*}(a, 0) \text { and } \Pi_{i}=\Pi_{i}^{*}(a, 0), i=0,1, \text { if } \bar{w} \in\left(w_{1}^{* *}, \bar{w}_{2}\right)
\end{aligned}
$$


(ii) $(a, b)$ and $\left(w_{0}, w_{1}\right)$ defined by $w_{0}^{*}(a, b)=w_{1}^{*}(a, b)=$

$\bar{w}=w_{0}=w_{1}, L_{i}=L_{i}^{*}(a, b)$ and $\Pi_{i}=\Pi_{i}^{*}(a, b), i=0,1$, if $\bar{w} \in\left[\bar{w}_{2}, \overline{\bar{w}}\right]$

(b) If $\delta=\left(0, \frac{1}{2}\right)$ and $\bar{w} \in(\overline{\bar{w}}, \beta]$, there is no pure strategy SPE.

Proof of Theorem 3 The proof involves a number of steps.

$\underline{\text { Step } 1}$ With reference to figure 4.1 and lemma 4.1 we have the following derivatives of $\Pi_{0}$ when $(a, b) \in T$.

(A) In region $\mathrm{A}, \Pi_{0}$ is firm 0's laissez-faire profit and $\partial \Pi_{0} / \partial a$ has the sign of $F(a, b)$ defined in the proof of lemma 3.3. In particular, since $\delta<\frac{1}{2}, \partial \pi_{0} / \partial a>0$ whenever $b>\sqrt{1-\delta}$.

(B) In region $\mathrm{B}, \partial \Pi_{0} / \partial a=\frac{1}{2}(\alpha-\bar{w})>0$

(C) In region $\mathrm{C}, \partial \Pi_{0} / \partial a$ has the sign of $\alpha-\bar{w}+t(1-a-b)(1-b-3 a)$. But in region C, $\bar{w}<\alpha-t\left[(1-b)^{2}-a^{2}\right]$, so $\alpha-\bar{w}>t(1-a-b)(1-b+a)$. It follows that $\partial \Pi_{0} / \partial a>0$ since $1-b+a>3 a+b-1($ i.e. $1>a+b)$.

(D) In region $\mathrm{D}, \partial \Pi_{0} / \partial a$ has the sign of $t(1-a-b)^{2}-(\beta-\bar{w})$. But in region $\mathrm{D}$, $\beta-\bar{w}>t(1-a-b)(1-a+b)>t(1-a-b)^{2}$, so $\partial \Pi_{0} / \partial a<0$

Step 2 The derivatives in Step 1 imply that, when $w_{1}^{* *} \leq \bar{w} \leq \bar{w}_{2}$, the unique (up to symmetry) remaining SPE candidate is at the point $P$ indicated in figure 4.2(a) and (b) on the border between regions $\mathrm{A}$ and $\mathrm{C}$. Since $b=0$ here and since Step 1 ensures that firm 0 will not want to deviate to any $a$ where $(a, 0) \in T, P$ is indeed SPE provided firm 0 's profit at $P$ is at least as large as at co-location $(a=1, b=0)$. This will be true if $H(a, \delta)=(1-a)\left(3+a+\frac{\delta}{1-a}\right)^{2}-18 \delta \geq 0 \quad$ everywhere on the set $\left\{(a, \delta): a \in\left[0, \frac{1}{2}\right], \delta \in\left[0, \frac{1}{2}\right]\right.$ and $\left.\delta \geq 2 a(1-a)\right\} . \quad$ It is straightforward to check that 
$\partial H / \partial \delta<0$ everywhere on this set. So the result will follow if $H\left(a, \frac{1}{2}\right) \geq 0$ for all $a \in\left[0, \frac{1}{2}\right], \quad$ or $\quad H(a)=[1+2(1-a)(3+a)]^{2}-36(1-a) \geq 0$ for $\quad a \in\left[0, \frac{1}{2}\right] \quad$ But $H(0), H\left(\frac{1}{2}\right)>0$ and $H$ is concave, ensuring the desired result and completing the proof of (a)(i).

Step 3 When $\bar{w}_{2} \leq \bar{w} \leq \overline{\bar{w}}$, the unique (up to symmetry) remaining SPE candidate is at $P$ in figure 4.2(c). Step 1 ensures that form 0 does not want to deviate from $P$ to any location which leaves $(a, b) \in T$.

We show next that firm 0 does not want to deviate from $P$ to collocate; this requires,

$$
\pi_{0}^{*}(a, b)=\frac{1}{18} t(1-a-b)\left[3+a-b+\frac{\delta}{1-a-b}\right]^{2} \geq \alpha-\beta
$$

where $\delta=2(1-a-b)(a-b)$. Equivalently,

$$
(1-a-b)\left[3+a-b+\frac{\delta}{1-a-b}\right]^{2} \geq 18 \delta=36(1-a-b)(a-b)
$$

which becomes $[1-(a-b)]^{2} \geq 0$, and clearly is satisfied. It remains to show that firm 0 does not want to deviate from $P$ to any location strictly to the right of firm 1 . For convenience let $\left(a^{*}, b^{*}\right)$ now denote $\mathrm{P}$; notice that $b^{*}<\frac{1}{2}$. From symmetry the profits attainable by firm 0 from such right deviations are the same as when firm 1 is at $1-b^{*}$ and firm 0 chooses strictly to the left of firm 1 . From step 1 the only candidates for local maxima of $\pi_{0}$ on this latter set are;

(a) along the $A / c$ border to the right of $P$

(b) in $A$ with $a=0$ if $\left(b^{*}<\right) 1-b^{*}<\sqrt{1-\delta}$.

The proof of (a) (ii) is completed by showing;

(i) along the $A / c$ border firm 0's profits decrease with $b$.

(ii) in $A$ where $a=0$ and $b<\sqrt{1-\delta}, \quad \partial \pi_{0}^{*} / \partial b<0$. 
For (i): here $\pi_{0}=\frac{1}{18} t(1-a-b)\left[3+a-b+\frac{\delta}{1-a-b}\right]^{2}$, $\bar{w}=w_{1}^{*}(a, b)=\frac{1}{3} \alpha+\frac{2}{3} \beta-\frac{1}{3} t(1-a-b)(3-a+b)$ so that $d a / d b=-(1+b)(2-a)^{-1}$, and $\delta<(1-a-b)(3-a+b)$

Differentiating $\pi_{0}$ totally with respect to $b$ and using the $d a / d b$ expression shows that $d \pi_{0} / d b<0$ if $d<(1-a-b)(9-a+b)$ which follows since $\delta<(1-a-b)(3-a+b)$

For (ii): it follows straightforwardly that, with $a=0, \partial \pi_{0}^{*} / \partial b<0$ iff $\delta /(1-b)<5-3 b$. Using the restriction $b<\sqrt{1-\delta}, \delta /(1-b)<1+b$ and the required inequality follows as $b<1$.

Finally, for $\bar{w}>\overline{\bar{w}}$ as in figure 4.2(d), Step 1 ensures that $P$ (the only candidate) is not an equilibrium completing (b).

When $\delta=0, \alpha=\beta, w_{1}^{* *}=\bar{w}_{1}=\bar{w}_{2}=\alpha-t$ and $\overline{\bar{w}}=\bar{w}_{3}=\alpha$. In this limit the arguments leading to Theorem 4, in particular part (a)(ii), can be applied to provide:

Corollary to Theorem 3 If $\delta=0$ and $\bar{w} \in\left(w_{1}^{* *}, \beta\right)$ the unique (up to symmetry) pure strategy SPE outcome has $a=b=\frac{1}{2}(1-(\alpha-\bar{w}) / t), L_{0}=L_{1}=\frac{1}{2} \quad$ and $\pi_{0}=\pi_{1}=\frac{1}{2}(\alpha-\bar{w})$ 


\section{SOCIAL WELFARE AND MINIMUM WAGES}

As $\bar{w}$ increases from $w_{1}^{* *}$ the SPE change as indicated in Theorem 3. In terms of figure 4.2 , as $\bar{w}$ increases from $w_{1}^{* *}$ to $\bar{w}_{2}$, the SPE locations have $b=0$ with a increasing from 0 to $\frac{1}{2}(1-\sqrt{1-2 \delta})$, as $\bar{w}$ increases from $\bar{w}_{2}$ to $\overline{\bar{w}}$, SPE locations follow the dotted curve $(\delta=2(1-a-b)(a-b))$ with $(a, b)$ increasing from $\left(\frac{1}{2}(1-\sqrt{1-2 \delta}, 0)\right)$ at $\bar{w}_{2}$ to $\left(\frac{1}{2}, \frac{1}{2}-\sqrt{\delta / 2}\right)$, and for $\bar{w}>\overline{\bar{w}}$ there is no pure strategy SPE. Theorem 4 below answers the question: what happens to social welfare in pure strategy SPE as $\bar{w}$ increases from $w_{1}^{* *}$ to $\overline{\bar{w}}$ ? Some preliminary points are;

i) All pure strategy SPE for $\bar{w} \in\left[w_{1}^{* *}, \overline{\bar{w}}\right]$, occur in (on the border of) region A, so the efficient firm market share is $L_{0}=\frac{1}{6}(3+a-b)+\delta / 6(1-a-b)$

ii)Let $S W(\bar{w})$ denote the value of social welfare in the pure strategy SPE at minimum wage $\bar{w} \in\left[w_{1}^{* *}, \overline{\bar{w}}\right]$, and let $S W\left(w_{1}^{* *}\right)=S W^{* *}$ denote its laissez-faire value.

iii)Let $S W^{\circ}$ denote the value of social welfare at the social optimum.

Theorem 4 Suppose $\delta \in\left(0, \frac{1}{2}\right)$ and $\bar{w} \in\left[w_{1}^{* *}, \overline{\bar{w}}\right]$.

(a) $\quad S W(\bar{w})$ is strictly increasing for $\bar{w} \in\left[w_{1}^{* *}, \bar{w}_{2}\right]$.

(b) If $\delta \in\left[\frac{2}{5}, \frac{1}{2}\right)$ then $S W(\bar{w})$ is strictly increasing for all $\bar{w} \in\left[w_{1}^{* *}, \overline{\bar{w}}\right]$

(c) If $\delta \in\left(0, \frac{2}{5}\right)$ there is a unique minimum wage, $\bar{w}^{*}$ say, which maximizes $S W(\bar{w})$ over $\bar{w} \in\left[w_{1}^{* *}, \overline{\bar{w}}\right]$; at $\bar{w}^{*}, a+b>\frac{1}{2}$.

(d) $\quad S W(\bar{w})>S W^{* *}$ for all $\bar{w} \in\left[w_{1}^{* *}, \overline{\bar{w}}\right]$.

(e) $\quad S W^{\circ}>S W(\bar{w})$ 


\section{Proof of Theorem 4}

First note that substitution of the laissez-faire SPE $\left(a=b=0, L_{0}=\frac{1}{2}+\frac{1}{6} \delta\right)$ into $S W(a$, $\left.b, L_{0}\right)$ produces, after some manipulation:

$$
t^{-1} S W^{* *}=\beta / t-\frac{1}{12}+\frac{1}{2} \delta+\frac{5}{36} \delta^{2}
$$

(a) For $\bar{w} \in\left[w_{1}^{* *}, \bar{w}_{2}\right]$, SPE locations are $b=0$ and $a=a(\bar{w})$, where $a(\bar{w})$ is defined by $\bar{w}=w_{1}^{*}(a, 0)=\frac{1}{3} \alpha+\frac{2}{3} \beta-\frac{1}{3} t(1-a)(3-a)$. It follows that

$a^{\prime}(\bar{w})=3 t^{-1}(4-2 \mathrm{a})^{-1}>0$. Social welfare can then be written as a function of $a$, on the domain where $a \in\left[0, \frac{1}{2}\right)$ and $\delta \geq 2 a(1-a)$;

$$
S W(a)=\alpha L_{0}+\beta\left(1-L_{0}\right)-\frac{1}{3} t\left[a^{3}+\left(L_{0}-a\right)^{3}+\left(1-L_{0}\right)^{3}\right]
$$

where $L_{0}=\frac{1}{6}(3+a)+\frac{\delta}{6(1-a)}$. Since $a^{\prime}(\bar{w})>0$ it suffices to show that $S W^{\prime}(a)>0$ on its domain. Differentiation and manipulation produces

$$
\begin{gathered}
36 t^{-1} S W^{\prime}(a)=4\left[1+\frac{\delta}{(1-a)^{2}}\right]\left[\delta+a-a^{2}\right]+\left[3+a+\frac{\delta}{1-a}\right]\left[3-11 a+\frac{\delta}{1-a}\right] \\
=5 \frac{\delta^{2}}{(1-a)^{2}}+10 \delta+9-15 a^{2}-26 a
\end{gathered}
$$

Since $\delta /(1-a) \geq 2 a$ on the domain,

$36 t^{-1} S W^{\prime}(a) \geq 9-6 a-15 a^{2}>0$ for $a \in\left[0, \frac{1}{2}\right)$, completing the proof of $(a)$.

$(b) /(c)$ For $\bar{w} \in\left(\bar{w}_{2}, \overline{\bar{w}}\right)$ SPE locations are $a(\bar{w})$ and $b=b(a)$ where $b(a)$ is defined by $\delta=2(1-a-b)(a-b)$ for $\left.a \in \frac{1}{2}(1-\sqrt{1-2 \delta}), \frac{1}{2}\right]$ and (e.g.) $a(\bar{w})$ is defined by $\quad \bar{w}=w_{1}^{*}(a, b(a))=\frac{1}{3} \alpha+\frac{2}{3} \beta-\frac{1}{3} t(1-a-b(a))(3-a+b(a))$.

Since

$b^{\prime}(a)=(1-2 a) /(1-2 b)$, it follows from $\bar{w}=w_{1}^{*}(a, b(a))$ that $a^{\prime}(\bar{w})=18 t^{-1}(1-2 b)^{-1}$

$(1-a-b)>0$. Again social welfare can be written as a function of a where $a \in\left(\frac{1}{2}(1-\sqrt{1-2 \delta}), \frac{1}{2}\right]$ 


$$
S W(a)=\alpha L_{0}+\beta\left(1-L_{0}\right)-\frac{1}{3} t\left[a^{3}+b^{3}+\left(L_{0}-a\right)^{3}+\left(1-b-L_{0}\right)^{3}\right]
$$

where $L_{0}=\frac{1}{2}(1-b+a)$ and $b=b(a)$. Since $a^{\prime}(\bar{w})>0$ the sign of $S W^{\prime}(\bar{w})$ coincides with that of $S W^{\prime}(a)$. Differentiation and manipulation produces

$$
t^{-1}(1-2 b) S W^{\prime}(a)=\delta(a-b)-a^{2}(1-2 b)-b^{2}(1-2 a)+\frac{1}{2}(1-a-b)^{3}
$$

Some manipulation and substitution of $a-b=\frac{1}{2} \delta /(1-a-b)$ produces:

$$
4 t^{-1}(1-s)^{2}(1-2 b) . S W^{\prime}(a)=\delta^{2}(3-5 s)-4(2 s-1)(1-s)^{3}=m(s) \text {, say, where } s
$$

$=a+b$.

It is easy to check that $m\left(\frac{1}{2}\right)>0, m^{\prime}(s)<0$ for $s \in\left[\frac{1}{2}, \frac{3}{5}\right]$ and $m(s)<0$ for $s \in\left(\frac{3}{5}, 1\right)$. It follows that $S W\left(\right.$ a) has a unique maximum on $\left(\frac{1}{2}\left(1-\sqrt{1-2 \delta}, \frac{1}{2}\right]\right.$, and is increasing (resp., decreasing) to the left (resp., right) of this value. When $a=\frac{1}{2}, b=\frac{1}{2}-\sqrt{\delta / 2}, s=1-\sqrt{\delta / 2}$ and $m(1-\sqrt{\delta / 2})$ has the sign of $5 \delta-2$. (b) and (c) now follow.

(d) From (a), (b) and (c) it suffices to show that $S W(\overline{\bar{w}})>S W^{* * *}$. At $\overline{\bar{w}}$, $a=\frac{1}{2}, b=\frac{1}{2}-\sqrt{\delta / 2}$ and the required inequality becomes after manipulation;

$$
n(y)=20 y^{3}-45 y^{2}+18 y-9<0
$$

where $y=\sqrt{\delta / 2} \in(0,1)$. Now $n(0)<0, n(1)<0, n$ is concave on $\left[0, \frac{3}{4}\right]$, convex on $\left[\frac{3}{4}, 1\right]$ A straightforward calculation shows that at the unique stationary point on $\left[0, \frac{3}{4}\right], n(y)<0$ which ensures the result.

(e) This follows since at the unique social optimum $a^{\circ}+b^{\circ}=\frac{1}{2}$ whereas at the SPE location which maximizes social welfare $a+b>\frac{1}{2}$.

Hence, as the minimum wage increases from $w_{1}^{* * *}$, the effect is to increase social welfare (up to the pure strategy existence limit $\bar{w}=\overline{\bar{w}}$ ) if $\delta \in\left[\frac{2}{5}, \frac{1}{2}\right)(\operatorname{part}(\mathrm{b}))$. If 
$\delta \in\left(0, \frac{2}{5}\right)$ the minimum wage (again up to the limit $\bar{w}=\overline{\bar{w}}$ ) always improves on laissez-faire (part (d)), but now the improvement is not monotonic over the whole $\bar{w}$ range (parts (a) and (c)), social welfare reaching a maximum at some $\bar{w}^{*} \in\left(w_{1}^{* *}, \overline{\bar{w}}\right)$. Although minimum wages (up to the pure strategy existence limit) always improve on laissez-faire, they never allow attainment of the full social optimum (part (e)).

On the other hand when $\delta=0, \alpha=\beta, w_{1}^{* *}=\bar{w}_{1}=\bar{w}_{2}=\alpha-t, \overline{\bar{w}}=\bar{w}_{3}=\alpha$, and the arguments of Theorem 4 produce instead:

Corollary to Theorem 4 If $\delta=0$ and $\bar{w} \in\left(w_{1}^{* *}, \beta\right)$ there is a unique minimum wage which maximizes $S W(\bar{w})$, namely $\bar{w}^{*}=\alpha-\frac{1}{2} t$, and now $S W\left(\bar{w}^{*}\right)=S W^{\circ}$; again $S W(\bar{w})>S W^{* * *}$ for all $\bar{w} \in\left(w_{1}^{* *}, \beta\right)$.

So with symmetric firm efficiency, minimum wages across the whole range $\left(w_{1}^{* *}, \beta\right)$ improve on laissez-faire, but the unique social welfare maximizing minimum wage now implements the full social optimum.

The above shows that in the "long-run", via its impact on the non-wage job characteristic, the imposition of a minimum wage is welfare-improving over laissezfaire. Finally we consider the "short-run" impact in which we assume that the nonwage job characteristic remains fixed at its laissez-faire level of $a=b=0$ or 1 (we continue to restrict attention to $\delta<1 / 2$ ). The short-run affect of a minimum wage is as follows, from Lemma 4.1;

(A) If $\bar{w} \leq w_{1}{ }^{* *}$ wages and market shares remain at the laissez-faire levels described in Theorem 1

(B) If $\alpha-t \leq \bar{w} \leq \beta$ the minimum wage binds on both firms $\left(w_{0}=w_{1}=\bar{w}\right)$ producing equal market shares 
(C) If $\mathrm{w}_{1}{ }^{* *} \leq \bar{w} \leq \alpha-t$ then $w_{0}=1 / 2(\alpha+\bar{w}-t), \mathrm{w}_{1}=\bar{w}$,

$$
\mathrm{L}_{0}=(\alpha-\bar{w}+t) / 4 \mathrm{t} \text { and } \mathrm{L}_{1}=1-\mathrm{L}_{0}
$$

Adapting the previous general formula, social welfare in the short run (SSW) depends on the minimum wage as follows:

$\operatorname{SSW}(\bar{w})=\alpha L_{0}+\beta\left(1-L_{0}\right)-1 / 3 \quad \mathrm{t}\left[L_{0}^{3}+\left(1-L_{0}\right)^{3}\right]$

Where $\mathrm{L}_{0}=\mathrm{L}_{0}(\bar{w})$ and;

$$
\begin{aligned}
& \mathrm{L}_{0}(\bar{w})=\mathrm{L}_{0}{ }^{* *} \text { if } \bar{w} \leq w_{1}{ }^{* *} \\
& \mathrm{~L}_{0}(\bar{w})=(\alpha-\bar{w}+t) / 4 t \text { if } \quad w_{1}^{* *} \leq \bar{w} \leq \alpha-t \\
& \mathrm{~L}_{0}(\bar{w})=1 / 2 \text { if } \alpha-t \leq \bar{w} \leq \beta
\end{aligned}
$$

When $\delta>0, \mathrm{w}_{1}{ }^{* *}<\alpha-\mathrm{t}$ and it is straightforward to check that the (continuous) function SSW is strictly decreasing then over the interval $\left[\mathrm{w}_{1}{ }^{* *}, \alpha-t\right]$, constant elsewhere. Thus;

Theorem 5 Suppose $\delta \varepsilon(0,1 / 2)$, then $\operatorname{SSW}(\bar{w})<\mathrm{SW}^{* *}$ for all $\bar{w} \varepsilon\left(w_{1}{ }^{* *}, \beta\right]$ And when $\delta=0, \operatorname{SSW}(\bar{w})$ is constant everywhere;

$\underline{\text { Corollary to Theorem } 5}$ If $\delta=0$ then minimum wages have no short-run effect on social welfare.

Thus in the short-run minimum wages typically $(\delta>0)$ reduce social welfare from the laissez faire level. What happens is that the minimum wage first binds only on the inefficient firm with the efficient firm responding by also increasing its wage from the laissez-faire level, but by less than the inefficient firm's (forced) increase. As a result the inefficient firm's market share increases, which causes the fall in welfare. Thus the positive long run welfare impact of minimum wages in Theorem 4 is driven by and dependent on the effect of minimum wages on the non-wage job characteristics. 


\section{6. $\quad \underline{\text { CONCLUSIONS }}$}

We have shown how the imposition of minimum wages can be welfare improving on laissez-faire, because of their impact on firms' choice of non-wage job characteristics. In the context of a Hotelling duopsony, jobs are horizontally differentiated (e.g. by location) and the effect of the minimum wage is to narrow the gap between "locations" chosen by firms compared to the maximum differentiation chosen under laissez-faire (at least when the efficiency differential between firms is small enough), in a welfare improving way. The paper thus provides a new route through which minimum wages can be "a good thing", over and above the beneficial employment effects of the previous literature. It also generates a natural question for further research in the differentiated oligopsony framework, namely the effect of minimum wages on vertically differentiated job characteristics (e.g. quality of the work environment), as opposed to our horizontal differentiation. 


\section{$\underline{\text { References }}$}

Bhaskar, V., and T. To (1999), "Minimum wages for Ronald McDonald monopolies: a theory of monopsonistic competition”, Economic Journal, 109, 190-203.

Bhaskar, V., and T. To (2003), "Oligopsony and the distribution of wages", European Economic Review, 47, 371-399.

d'Aspremeont, C., J-J. Gabszewicz and J-F. Thisse (1979), "On Hotelling's stability in competition", Econometrica, 47, 1145-1150.

Lederer, P.J. and A.P. Hurter(1986), "Competition of firms; discriminatory pricing and location", Econometrica, 53(3), 623-640.

Manning, A. (2003), Monopsony in Motion, Princeton University Press, Princeton.

Ziss, S. (1993), "Entry deterrence, cost advantage and horizontal product differentiation", Regional Science and Urban Economics, 23, 523-543. 


\section{$\underline{\text { APPENDIX }}$}

Proof of Lemma 3.1 (a) From the definitions of $\Pi_{0}$ and $\tilde{x}$;

(1) $\Pi_{0}=0$ if $\quad \tilde{x} \leq 0$, i.e. $w_{0} \leq w_{1}-t\left[(1-b)^{2}-a^{2}\right]$

(2) $\Pi_{0}=\left(\alpha-w_{0}\right) \tilde{x} \quad$ if $\quad \tilde{x} \in(0,1]$, i.e. $w_{1}-t\left[\left(1-b^{2}-a^{2}\right]<w_{0} \leq w_{1}+t\left[(1-a)^{2}-b^{2}\right]\right.$

(3) $\Pi_{0}=\alpha-w_{0}$ if $\quad \tilde{x} \geq 1$, i.e. $w_{1}+t\left[(1-a)^{2}-b^{2}\right] \leq w_{0}$

It is easy to check that (1), (2) and (3) define $\Pi_{0}$ as a continuous, quasi-concave function of $\mathrm{w}_{0}$ over the whole range $[0, \alpha]$ (constant at 0 over the range of (1), strictly concave over (2) and linear, decreasing over (3)).

If $w_{1} \geq \Lambda_{1}$ then $\tilde{x} \leq 0$, and so $L_{0}=\Pi_{0}=0$, for all $w_{0} \in[0, \alpha]$. Thus any $w_{0} \in[0, \alpha]$ is a best response for firm 0 to $w_{1} \geq \Lambda_{1}$. If $w_{1}<\Lambda_{1}$ then strictly positive profits are attainable by firm 0 (by choosing $w_{0}=\alpha-\varepsilon, \varepsilon$ small enough), and a best response must lie in the range of (2) above. In this range, $\Pi_{0}$ is a strictly concave function of $w_{0}$ with stationary point $w_{0}=\frac{1}{2}\left\{\alpha+w_{1}-t\left[(1-b)^{2}-a^{2}\right]\right\}$ which lies in the range of (2), and so is the best response, iff $w_{1} \in\left[\gamma_{1}, \Lambda_{1}\right)$. If $w_{1}<\gamma_{1}, \Pi_{0}$ is increasing over the range of (2) so the maximum of $\Pi_{0}$ occurs at $w_{0}=w_{1}+t\left[(1-a)^{2}-b^{2}\right]$, which is therefore the best response. Interchanging $0 / 1$ subscripts, $a / b$ and $\alpha / \beta$, and replacing $\tilde{x}$ by $(1-\tilde{x})$ produces the firm 1 result. Thus the set of subgame NE for $(a, b) \in S$ correspond to simultaneous solutions of one of (i) - (iii) in (a) with one of (iv) - (vi), where $w_{0} \in[0, \alpha]$ and $w_{1} \in[0, \beta]$

(b) Assume $(a, b) \in T$ and consider the (ii)/(v) pairing. The equations intersect at $w_{i}=w_{i}^{*}(a, b), i=0,1$ and the resulting $w_{0}, w_{1}$ satisfy the inequalities in (ii)/(v) iff $\delta<(1-a-b)(3-a+b)($ or $(a, b) \in T)$. It is straightforward to check that no pairings 
produce any other NE for $(a, b) \in T$, which completes the proof of (b), using the NE wages to derive the corresponding market shares and profits.

(c) Assume $(a, b) \in S / T$. Consider the (i)/(vi) pairing where $w_{1}=\beta$ in (vi). The resulting wages $\left(w_{0}=\beta+t\left[(1-a)^{2}-b^{2}\right], w_{1}=\beta\right)$ satisfy the required inequalities iff $\delta>(1-a-b)(3-a+b)$. The (ii)/(vi) pairing with $w_{1}=\beta$ produces $w_{0}=\frac{1}{2}\left\{\alpha+\beta-t\left[(1-b)^{2}-a^{2}\right]\right\}, \quad w_{1}=\beta$ which satisfies the inequalities iff $\delta=(1-a-b)(3-a+b)$, in which case $w_{0}=\beta+t\left[(1-a)^{2}-b^{2}\right]$. Again one can check that no pairings produce other NE for $(a, b) \in S / T$ and that the NE wages produce the market shares and profits in (c). Consider now the case $(a, b) \in H$. From the definition of $L_{0}$ for this case, if $w_{1}=\beta$, firm 0 attains $\Pi_{0}=\alpha-\beta>0$ with $w_{0}=\beta$, which cannot be improved upon $\left(w_{0}>\beta \Rightarrow \Pi_{0}=\alpha-w_{0}<\alpha-\beta, w_{0}<\beta \Rightarrow \Pi_{0}=0\right)$. If $w_{0}=\beta$, firm 1 can do no better than choose $w_{1}=\beta$ giving $\Pi_{1}=0$. Thus $w_{0}=w_{1}=\beta$ is a NE. This is the unique NE: if $w_{1}<\beta$ then $w_{0}=w_{1}$ is again 0 's best response giving $\Pi_{1}=0$, but $w_{1}+\varepsilon(<\beta, \varepsilon>0)$ strictly improves for 1 ; market shares and profits are as claimed, completing the proof.

\section{$\underline{\text { Proof of Lemma } 3.3}$}

Suppose $0<\delta \leq \delta^{*}$. From lemma 3.1 we have;

(i) $\partial \Pi_{0}^{*} / \partial a=2 t(1-a)>0$ when $(a, b) \in(S \cup H) \backslash T$ and $a<1$.

(ii) When $(a, b) \in T, \partial \Pi_{0}^{*} / \partial a=\frac{1}{18} t\left(\frac{\delta}{1-a-b}+3+a-b\right)\left(\frac{\delta}{1-a-b}-1-3 a-b\right)$ whose sign coincides with that of $F(a, b)=\delta-(1-a-b)(1+3 a+b)$. The curve $F(a, b)$ $=0$ intersects the boundary of $T$ where $\delta=(1-a-b)(3-a+b)$ uniquely at $a=\frac{1}{2}$, $b=\frac{1}{2} \sqrt{9-4 \delta}-1$, the boundary of $T$ where $a=0$ uniquely at $b=\sqrt{1-\delta}$, and is 
downward sloping in $T$ between these intercepts when $\delta \leq \frac{3}{4}$. For $\delta \in\left(\frac{3}{4}, \delta^{*}\right]$, the curve slopes down when $3 a+2 b>1$, but is upward sloping when $3 a+2 b<1$ (with a turning point at $\left.b=2-\sqrt{3 \delta}, a=\frac{1}{3}(1-2 \mathrm{~b})\right)$. In each case, $\partial \Pi_{0}^{*} / \partial a>0$ to the right of the curve and $\partial \Pi_{0}^{*} / \partial a<0$ to the left.

Consider 0's constrained best response problem: $\max _{a} \Pi_{0}^{*}(a, b)$ s.t. $a \in[0,1-b]$. Define $G(\mathrm{~b}, \delta)=\Pi_{0}^{*}(0, b)-\Pi_{0}^{*}(1-b, b)$ on the domain $b \in[0, \sqrt{1-\delta}], \delta \in\left(0, \delta^{*}\right]$. Then;

$G(b, \delta)=\frac{1}{18} t\left[(1-b)\left(\frac{\delta}{1-b}+3-b\right)^{2}-18 \delta\right]$,

$\partial G / \partial b=\frac{1}{18} t\left(\frac{\delta}{1-b}+3-b\right)\left(\frac{\delta}{1-b}+3 b-5\right) \leq \frac{1}{18} t\left(\frac{\delta}{1-b}+3-b\right)(4 b-4)<0$

and $\partial G / \partial \delta=\frac{1}{9} t\left(\frac{\delta}{1-b}-b-6\right)<0\left(\operatorname{using} \frac{\delta}{1-b} \leq 1+b\right.$ on the domain $)$.

Thus there is a decreasing function $b(\delta)$ on the domain $\delta \in\left(0, \delta^{*}\right]$ such that $b=b(\delta)$ iff $G(b, \delta)=0, b<b(\delta)$ iff $G(b, \delta)>0$ and $b>b(\delta)$ iff $G(b, \delta)<0$. Moreover $\lim _{\delta \rightarrow 0} b(\delta)=1$ (since $G(1, \delta) \rightarrow 0 \quad$ as $\delta \rightarrow 0), \quad b\left(\frac{1}{4}\right)=\frac{1}{2} \quad\left(\right.$ since $\left.G\left(\frac{1}{2}, \frac{1}{4}\right)=0\right) \quad$ and $\quad b\left(\delta^{*}\right)=0$ (since $G(0, \delta)=\frac{1}{18} t\left[(\delta+3)^{2}-18 \delta\right]=0$ when $\left.\delta=\delta^{*}\right)$.

In the case where $\delta \in\left(0, \frac{3}{4}\right]$, the derivative signs in (i) and (ii), and the downward slope of the curve $F(a, b)=0$ imply that $a=0$ and $a=1-b$ are the only 2 candidates for 0 's constrained best response when $b \in[0, \sqrt{1-\delta}]$, and it follows from the previous paragraph that $a=0$ if $b<b(\delta), a=\{0,1-b\}$ if $b=b(\delta)$ and $a=1-b$ if $b>b(\delta)$. Moreover $\Pi_{0}^{*}(0, b)=\frac{1}{18} t(1-b)\left(\frac{\delta}{1-b}+3-b\right)^{2}$ is continuous and strictly decreasing in $\mathrm{b}$, and $\Pi_{0}^{*}(1-b, b)=\alpha-\beta$ independent of $b$. When $\delta \in\left(0, \frac{1}{4}\right), b(\delta)>\frac{1}{2}$ and using the 
symmetry of the $(a, b)$ and $(1-a, 1-b)$ subgames, 0 's unconstrained best response is as described in (a). When $\delta \in\left[\frac{1}{4}, \frac{3}{4}\right], b(\delta) \leq \frac{1}{2}$ and the symmetry ensures the unconstrained best response of (b).

When $\delta \in\left(\frac{3}{4}, \delta^{*}\right]$, the above arguments ensure the unconstrained best responses in (b) if $b \in[0, \sqrt{1-\delta}]$ or if $b \in[2-\sqrt{3 \delta}, 1]$. When $b \in(\sqrt{1-\delta}, 2-\sqrt{3 \delta})$ the candidates for 0 's constrained best response are $a=1-b$ and the value of a where $(a, b) \in T$ is on the upward sloping part of the $F(a, b)=0$ curve; let $a=a(b)$ denote this curve, defined by $F(a, b)=0$ and $3 a+2 b<1$ for $b \in(\sqrt{1-\delta}, 2-\sqrt{3 \delta})$. Along this curve 0 's profit is $\Pi_{0}^{*}(a(b), b)$ whose derivative with respect to $b$ is $-4(1-a-b)<0$. Also $G(\sqrt{1-\delta}, \delta)=\frac{1}{9} t(8-9 \delta-8 \sqrt{1-\delta})<0$ so $b(\delta)<\sqrt{1-\delta}$, and $a=1-b$ is 0 's constrained best response to any $b>b(\delta)$, as in the last paragraph, producing again the (b) statement.

Proof of Theorem 2 First note the following features of $f(a, b)$.

(i) $\frac{1}{t} \frac{\partial f}{\partial a}=\frac{1}{2} \delta^{2} \ell^{-2}+\frac{1}{2} \delta-a^{2}-\frac{1}{8}\left(\delta \ell^{-1}+\ell\right)^{2}\left(\delta \ell^{-2}-1\right)+\frac{1}{8}\left(\ell-\delta \ell^{-1}\right)^{2}\left(\delta \ell^{-2}+1\right)$

$$
=\frac{1}{2} \delta-a^{2}+\frac{1}{4} \ell^{2}+\frac{1}{4} \delta^{2} \ell^{-2}
$$

(ii) $\frac{1}{t} \frac{\partial f}{\partial b}=-\frac{1}{2} \delta-b^{2}+\frac{1}{4} \ell^{2}+\frac{1}{4} \delta^{2} \ell^{-2}$

(iii) Equating (i) and (ii) to $0, f$ has a unique stationary point $a=\frac{1}{4}+\delta, b=\frac{1}{4}-\delta$ with $\quad f(a, b)=f^{*}=\alpha-\frac{1}{2} \delta t+\delta^{2} t-\frac{1}{48} t$. Now consider problem 1: $\max _{(a, b)} g(a, b)$ s.t. $\delta \geq(1-a)^{2}-b^{2},(a, b) \in S \cup H . \quad$ The $\quad$ solutions $\quad$ are $\quad a=\frac{1}{2}$, $b \in\left[\sqrt{\frac{1}{4}-\delta}, \frac{1}{2}\right]$ if $\delta<\frac{1}{4}$, and $a=\frac{1}{2}, \quad b \in\left[0, \frac{1}{2}\right]$ if $\delta \geq \frac{1}{4}$; in both cases the optimal value 
is $g^{*}=\alpha-\frac{1}{12} t$. If $\delta \geq 1$, the feasible set for problem 1 is $S \cup H$ and the solution to problem 1 is then necessarily the social optimum.

Suppose $\delta<1$ from now on.

Next consider problem 2: $\max _{(a, b)} f(a, b)$ s.t. $\delta \leq(1-a)^{2}-b^{2},(a, b) \in S \cup H$. The feasible set is nonempty (with a non-empty interior) and compact, so there is a solution. But solutions cannot occur,

(1) on the feasible set boundary where $a=0, b \in[0, \sqrt{1-\delta})$ since $t^{-1} \partial f / \partial a=\frac{1}{4}\left(\frac{\delta}{1-b}+1-b\right)^{2}>0$

(2) on the feasible set boundary where $b=0, a \in[0,1-\sqrt{\delta})$ since $t^{-1} \partial f / \partial b=\frac{1}{4}\left(1-a-\frac{\delta}{1-a}\right)^{2}>0$ there.

In addition, when $\delta \geq \frac{1}{4}$ the (unique) stationary point is not interior to the feasible set, so any solution to problem 2 belongs to the boundary where $\delta=(1-a)^{2}-b^{2}$ and $a, b \geq 0$. But $f$ and $g$ coincide on this boundary which was also feasible, but not optimal, in problem 1. It follows that the solution to problem 1 provides the social optimum for all $\delta \geq \frac{1}{4}$, completing (b). Finally, when $\delta<\frac{1}{4}$, the stationary point of $f$ is interior to the feasible set of problem 2 with value $f^{*}$; moreover $f^{*}>g^{*}$ then. Thus the (unique) stationary point is the only solution candidate interior to the feasible set for problem 2, and there cannot be a boundary solution. So the stationary point solves problem 2 and, since $f^{*}>g^{*}$, provides the social optimum; hence (a). 


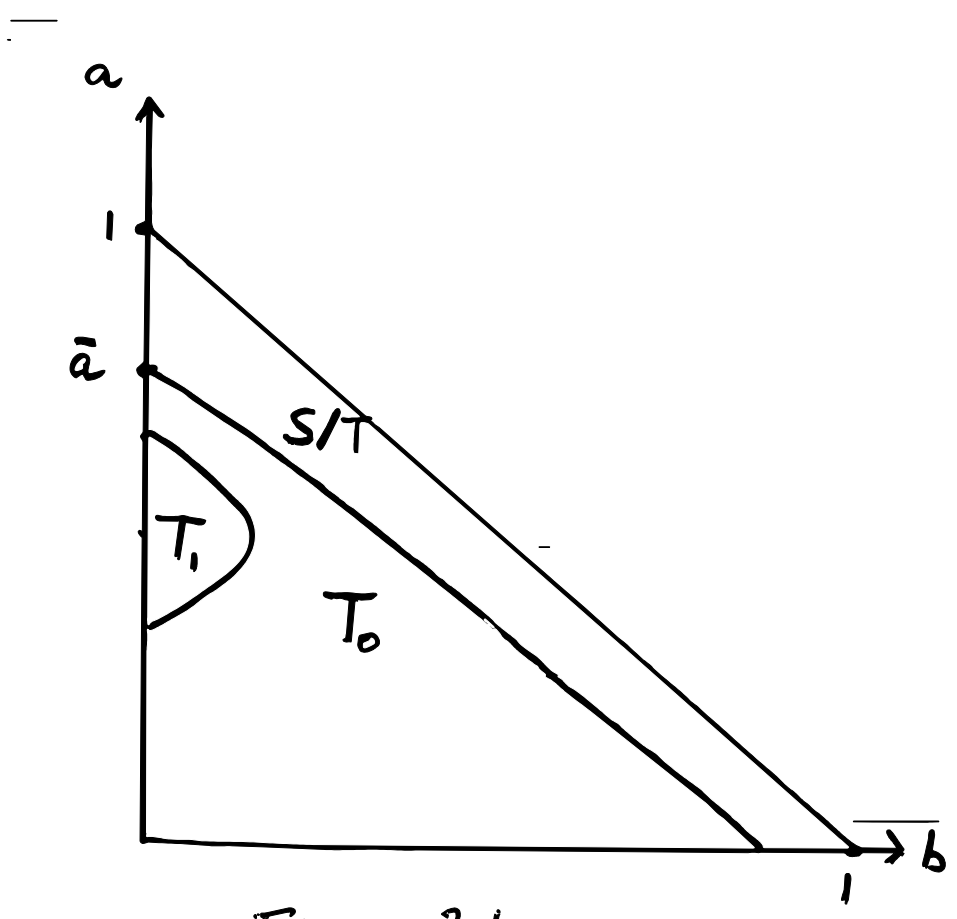

Figure 3.1

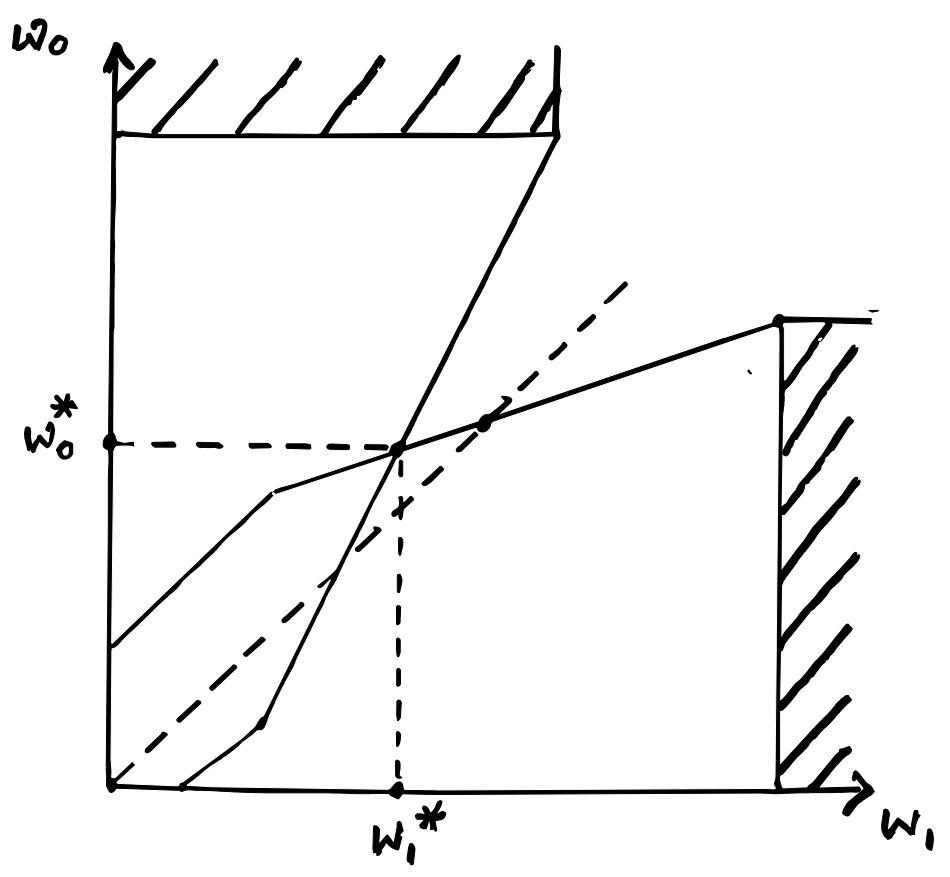

Figure 3.2 

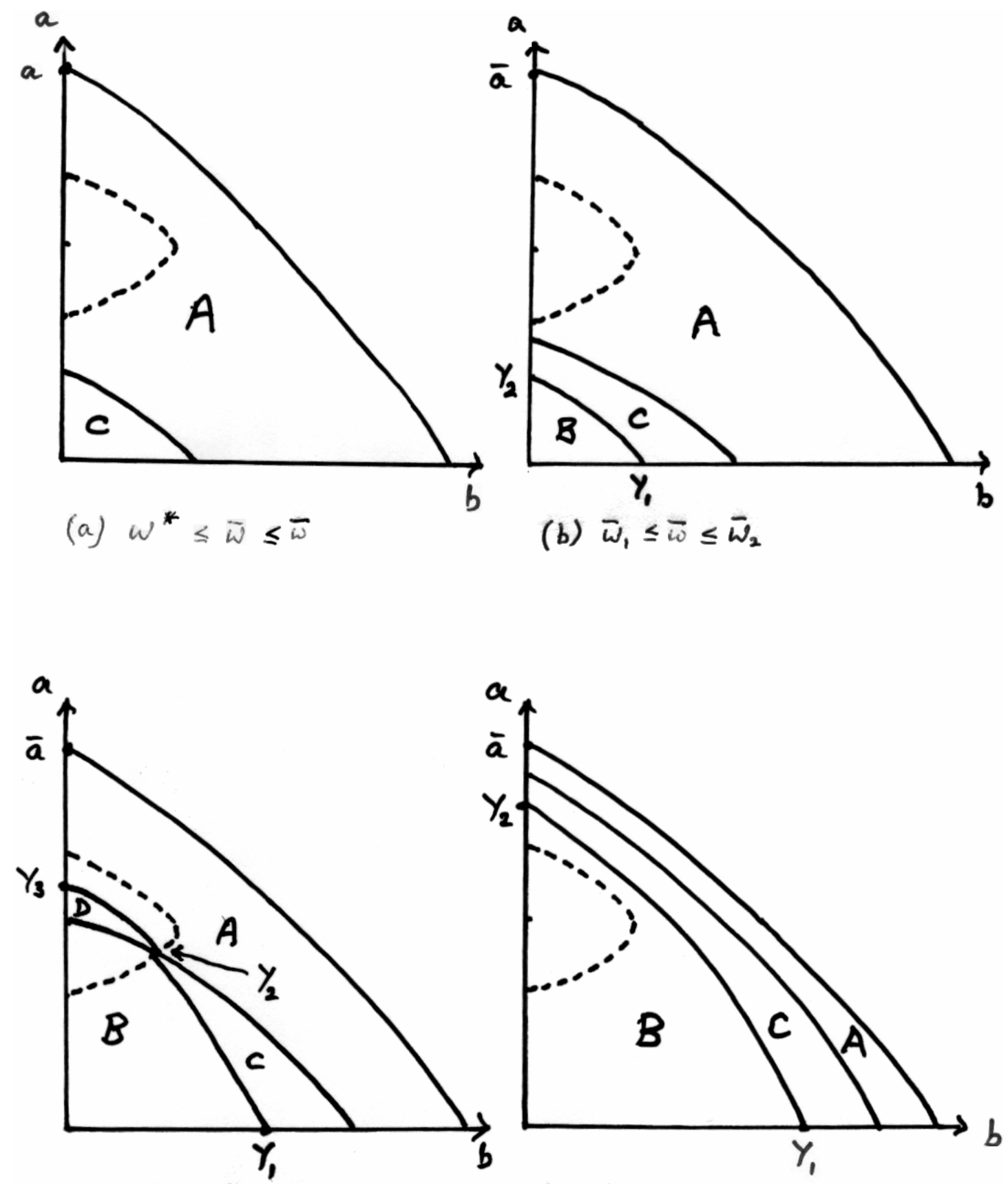

(c) $\bar{w}_{2} \leq \bar{w} \leq \bar{w}_{3}$

(d) $\bar{w}_{3} \leq \bar{w} \leq \beta$

Figure 4.1 


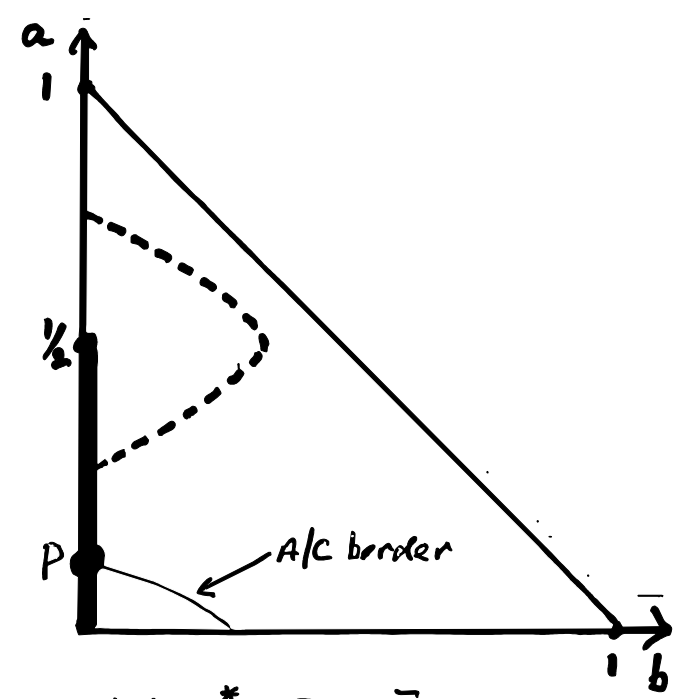

(a) $\omega_{1}^{*} \leq \bar{\omega} \leq \bar{\omega}$

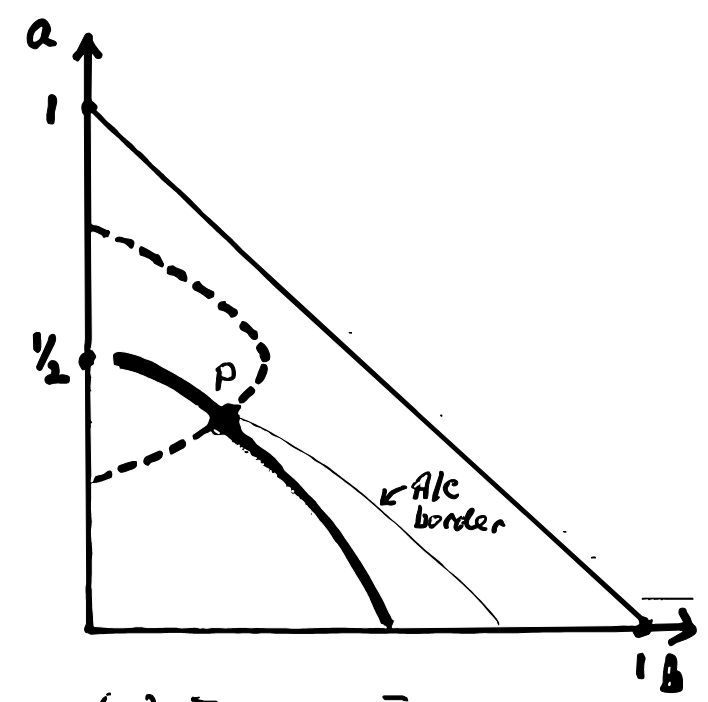

(c) $\bar{\omega}_{2} \leq \bar{\omega} \leq \overline{\bar{\omega}}$
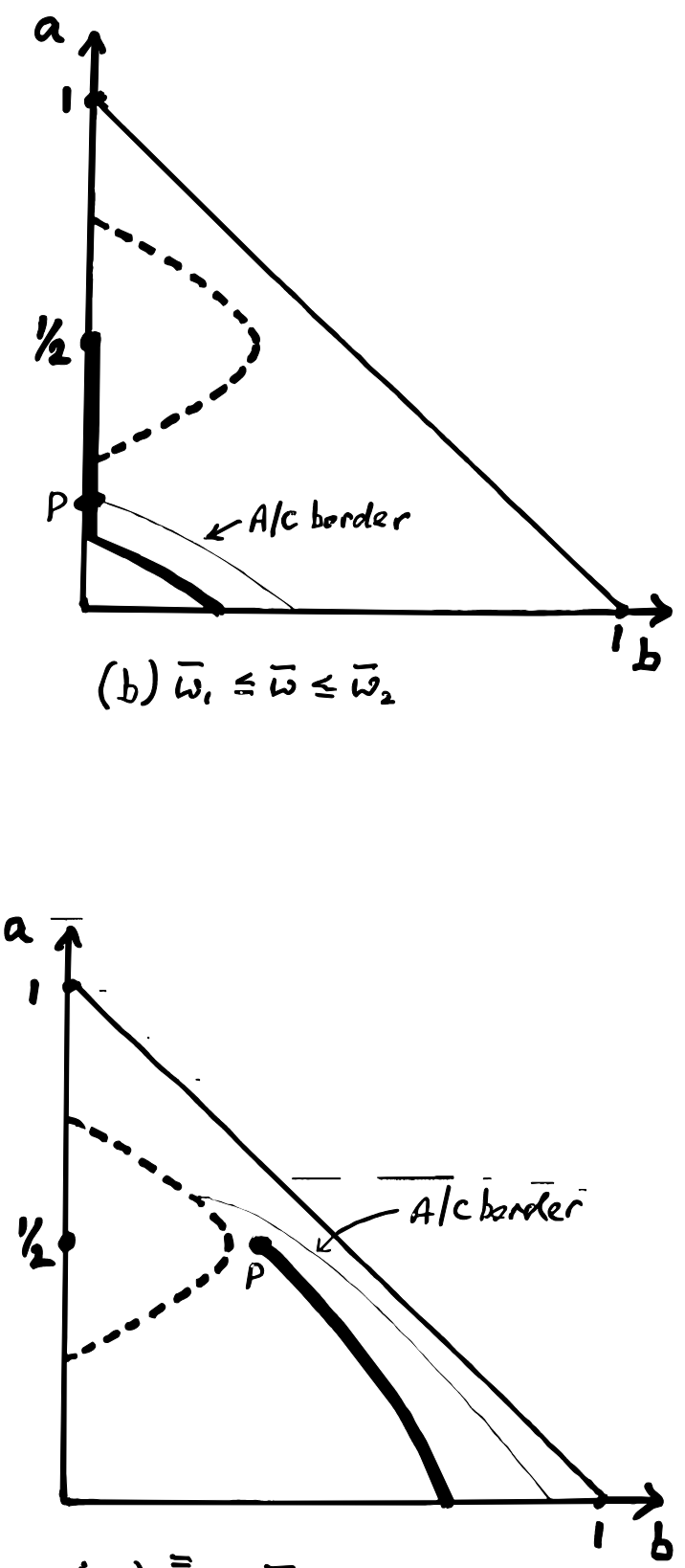

(d) $\bar{\omega}<\bar{\omega}$

Figure $4 . \overline{2}$ 